\title{
Temporal evolution and instability in a viscoelastic dielectric elastomer
}

\section{Citation}

Kollosche, Matthias, Guggi Kofod, Zhigang Suo, and Jian Zhu. 2015. “Temporal Evolution and Instability in a Viscoelastic Dielectric Elastomer." Journal of the Mechanics and Physics of Solids 76 (March): 47-64. doi:10.1016/j.jmps.2014.11.013.

\section{Published Version}

doi:10.1016/j.jmps.2014.11.013

\section{Permanent link}

http://nrs.harvard.edu/urn-3:HUL.InstRepos:13910327

\section{Terms of Use}

This article was downloaded from Harvard University's DASH repository, and is made available under the terms and conditions applicable to Open Access Policy Articles, as set forth at http:// nrs.harvard.edu/urn-3:HUL.InstRepos:dash.current.terms-of-use\#OAP

\section{Share Your Story}

The Harvard community has made this article openly available.

Please share how this access benefits you. Submit a story.

Accessibility 


\title{
Temporal evolution and instability in a viscoelastic dielectric elastomer
}

\author{
Matthias Kollosche a, Guggi Kofod ${ }^{b}$, Zhigang Suo ${ }^{\mathrm{c}}$, Jian Zhu d, * \\ a Applied Condensed-Matter Physics, Institute of Physics and Astronomy, Faculty of \\ Science, University of Potsdam, 14476 Potsdam-Golm, Germany \\ b InMold Biosystems A/S, 2630 Taastrup, Denmark \\ c School of Engineering and Applied Sciences, Kavli Institute for Bionano Science and \\ Technology, Harvard University, Cambridge, MA o2138, USA \\ d Department of Mechanical Engineering, National University of Singapore, 117575, Singapore
}

\begin{abstract}
Dielectric elastomer transducers are being developed for applications in stretchable electronics, tunable optics, biomedical devices, and soft machines. These transducers exhibit highly nonlinear electromechanical behavior: a dielectric membrane under voltage can form wrinkles, undergo snap-through instability, and suffer electrical breakdown. We investigate temporal evolution and instability by conducting a large set of experiments under various prestretches and loading rates, and by developing a model that allows viscoelastic instability. We use the model to classify types of instability, and map the experimental observations according to prestreches and loading rates. The model describes the entire set of experimental observations. A new type of instability is discovered, which we call wrinkle-to-wrinkle transition. A flat membrane at a critical voltage forms wrinkles and then, at a second critical voltage, snaps into another state of winkles of a shorter wavelength. This study demonstrates that viscoelasticity is essential to the understanding of temporal evolution and instability of dielectric elastomers.
\end{abstract}

Keywords: Dielectric elastomer, Viscoelasticity, Snap-through instability, Phase transition, Wrinkling.

*Corresponding author. Email: mpezhuj@nus.edu.sg (Jian Zhu) 


\section{Introduction}

The ability to control motion by means of electricity has been sought ever since the scientific dispute on the origin of electricity and muscular contraction in a dissected frog (Galvani, 1791; Volta, A. 1918). The dispute is often recognized as an opening for the physical understanding of modern electricity and the development of the modern battery by Volta (Volta, 1800). Many devices and systems capable of voltage-induced motion have been developed since then. However, only few display the unique inherent properties of soft viscoelastic response and large deformation found in biological systems.

First reported in 1880 by Röntgen (1880), the dielectric elastomer actuator is one particular type of actuator, which relies on the electric field to induce large deformation (Pelrine et al., 2000). A dielectric elastomer actuator consists of an elastomeric membrane sandwiched between two compliant electrodes (Fig. 1). When the electrodes are subject to a voltage, electrical charges of the opposite polarities accumulate on the two electrodes, causing the dielectric membrane to reduce thickness and increasing area. This electromechanical transduction has many desirable attributes, including large voltage-induced deformation, high energy density, fast response, quiet operation, light weight, and low cost (Pelrine et al., 2000; Ashley, 2003; Carpi et al., 2010). Dielectric elastomers are being employed for interesting applications, including tuning stage for soft diffractive grating (Aschwanden and Stemmer, 2006; Kollosche et.al. 2011), tunable organic laser (Döring et al., 2011), energy harvesters (Koh et al., 2011; Ahnert et al., 2011; McKay et al., 2011; Kornbluh et al., 2011;), soft motors (Anderson et al., 2011; O’Brien et al., 2012), soft computers (O’Brien et.al., 2013), structural health monitoring for buildings (Kollosche et al., 2011; Laflamme et al., 2010). In spite of these promising applications of soft transducers, the interplay of mechanics, electrostatics and designs in dielectric elastomers remains challenging.

The operation of dielectric elastomers is limited by electromechanical instability (EMI). Stark and Garton (1955) applied an electrical field to irradiated polyethylene under temperature 
variation, and found that EMI is responsible for electrical failure of this deformable polymer. EMI in dielectric elastomer is a positive feedback mechanism. When the voltage increases slightly, actuation causes thinning of the membrane, inducing even higher electric field. Due to the non-linear mechanical properties of elastomer, the positive feedback may cause the dielectric elastomer to thin down drastically, resulting in electrical failure (Zhao et al., 2007; Kollosche \& Kofod, 2010). EMI can be averted by affecting the non-linear properties of the elastomer, for instance by prestretching (Pelrine et al., 2000; Koh et al., 2011; Huang et al., 2012), or by employing interpenetrating networks (Ha et al., 2006; Suo and Zhu, 2009). Alternatively, control of charge instead of voltage provides a robust method of averting EMI (Keplinger et al., 2010; Li et al., 2011), but this control is only effective if the dielectric is actuated without electrodes, or when the electrodes have small areas ( $\mathrm{Lu}$ et al., 2014). In addition, there are conditions of particular boundary constraints under which EMI can be harnessed to achieve giant voltage-induced deformation (Zhao and Suo, 2010; Kollosche et al., 2012), including coupling to a pressurized container leading to planar actuation strain of more than $1600 \%$ through an aneurismic instability (Keplinger et al., 2012; Li et al., 2013). These previous papers disregarded the viscoelastic properties of the elastomer, and focused on their nonlinear stress-strain properties. However, we will later show that these effects must be included for a fuller understanding.

A membrane of a dielectric elastomer may form wrinkles as the applied voltage ramps up. During what we term the flat-to-wrinkled (FW) transition, the homogeneous flat state of the dielectric elastomer becomes unstable, giving way to an inhomogeneous state in which the flat and wrinkled regions may coexist in the membrane (Plante and Dubowsky, 2006; Zhou et al., 2008; Huang and Suo, 2011; Zhu et al., 2012). Recently, we investigated two types of FW transition in a clamped membrane (Zhu et al., 2012). During type I FW transition, nucleation of wrinkles occur in several small regions, followed by growth of each region until the whole membrane has undergone the transition. During type II FW transition, wrinkles form globally 
throughout the flat membrane, without the presence of a nucleation energy barrier. These two types of FW transition are analogous to the first and the second order phase transitions. While the type I FW transition is discontinuous and accompanied by a jump in deformation, the type II FW transition is accompanied by a continuous change in the deformation. Keplinger et al. (2008) reported a slow viscoelastic drift in the FW transition. In that experiment, the dielectric elastomer was subject to stepwise voltages. It was found that at a certain voltage, wrinkled regions may grow, slowly driving the dielectric elastomer into failure.

Subject to electromechanical loads, a dielectric elastomer may exhibit time-dependent response. For example, a number of experiments with spectacular results were performed on a particular elastomeric material, known as VHB (Very-High-Bonding, 3M). This elastomer has a relatively high dielectric constant, a very large elastic stretchability, and sufficient breakdown strength to enable voltage-induced strain above 100\% (Pelrine et al., 2000; Ha et al., 2006; Keplinger et al., 2012; Kollosche et al., 2012). Importantly, VHB exhibits large viscoelastic effects, and experiments showed that the time for its viscoelastic relaxation is several hundred seconds (Wissler et al., 2007, Carpi et al., 2008; Keplinger et al., 2008; Zhao et al., 2011).

Viscoelasticity determines the temporal properties of polymers. The viscoelastic behavior of elastomers is derived from their network structure. Each polymer chain is connected to its neighbors by covalent crosslinks, allowing a chain to twist and coil, and neighboring chains to entangle. When the elastomer deforms, polymer chains will rearrange through localized slipping and sliding, leading to viscoelastic relaxation of mechanical stresses.

Plante and Dubowsky (2007) employed an electromechanical model with an exponentially decaying time response to analyze the strain rate effect on stress-strain curves. Wissler and Mazza (2007) attempted to describe time dependence similarly, using the Prony series approach based on multiple exponentially decaying components. This approximate method results from assuming time-dependent coefficients in the strain energy functions. However, the theoretical prediction does not match experimental results well, especially when 
the voltage is large. Recently, more sophisticated viscoelastic models have been proposed to analyze the behavior of dielectric elastomers (Hong, 2011; Zhao et al., 2011; Foo et al., 2012; Park and Nguyen, 2013). However, these theories are compared with only few experimental results, so that the impact of viscoelasticity on the electromechanical behavior of a dielectric elastomer actuator is still unclear and deserves further investigation.

In this paper, we use a relatively simple setup of a dielectric elastomer actuator, a membrane prestretched by rigid clamps and a mechanical force (Fig. 2). As previously discussed (Kollosche et al., 2012), this setup enables control and measurement of the two independent variables voltage $\Phi$ and load $P$. Here, the rigid clamps and applied force prescribe well-defined prestretches in planar directions, $\lambda_{1 p}$ and $\lambda_{2 p}$. We vary the two prestretches systematically over a large range. The viscoelastic response is investigated by varying the voltage ramp rate for each individual set of prestretches. This paper studies in details the interplay among electromechanical behaviour, viscoelastic properties and instability effects, showing for the first time how control of voltage ramp rate can directly switch between different types of instability. The meticulous experimental investigations enable the discovery of a new type of instability, which we term wrinkle-to-wrinkle (WW) transition. A flat membrane forms wrinkles at a critical voltage. Then, at a higher voltage, the membrane snaps into another state of wrinkles of a shorter wavelength, accompanied by a jump in the displacement in the direction of the applied force.

In addition, we propose a method to analyze the snap-through instability by using a model that incorporates viscoelasticity. Both theory and experiments show that viscoelasticity significantly affects the electromechanical behavior of a dielectric elastomer. For example, with intermediate prestretches (say $\lambda_{1 p}=\lambda_{2 p}=4$ in Fig. 2), the membrane will exhibit a continuous FW transition at a small voltage ramp rate (say, $1 \mathrm{~V} / \mathrm{s}$ or $5 \mathrm{~V} / \mathrm{s}$ ), but exhibit a discontinuous WW transition at a large voltage ramp rate (say, $50 \mathrm{~V} / \mathrm{s}$ or $100 \mathrm{~V} / \mathrm{s}$ ). Furthermore, the comparison 
between theory and experiments show that VHB is still a predictable viscoelastic material, which can undergoes snap-through instability and achieve large voltage-induced deformation.

This paper is organized as follows. The experimental results are shown in Section 2. The theoretical modeling is developed in Section 3. Section 4 discusses a discontinuous FW transition. Section 5 analyzes a new type of WW transition. Effects of viscoelasticity on electromechanical behavior of a dielectric elastomer are studied in Section 6. All results are summarized in Section 7.

\section{Experimental observations}

The observations on clamped dielectric elastomers are obtained using an experimental setup sketched in Fig. 2-1. This setup consists of a high voltage generator (HCP $35-3500$, FUG Elektronik $\mathrm{GmbH}$ ) linked to a mechanical tensile tester (Zwick Roell Zoo5, equipped with a 10N load cell), controlled by a self-developed tuning procedure within the PC software (TestExpert II, Zwick Roell GmbH). The tensile tester applies the mechanical boundary conditions, while the generator applies a voltage drop. The control algorithm allows for continuous tracking of voltage induced deformation under constant force condition by feedback-controlled re-positioning of sample extension in the vertical direction.

The freestanding linear actuators are fabricated as follows. A stress-free membrane (VHB 4905), illustrated in Fig. 2-2, is of thickness $\mathrm{H}(=0.5 \mathrm{~mm})$, length $L_{1}$, and width $L_{2}$. The membrane is then attached to a mechanical stretching device and strained initially in both horizontal and vertical directions (Fig. 2-3). Stiff plastic frames are glued to the membrane to fix the horizontal prestretch $\lambda_{2}$, and two narrow strips of VHB9460 with a thickness of $50 \mu \mathrm{m}$ are attached to reinforce the edges to avoid cracks running into the clamped membrane. Compliant electrodes made of conductive carbon black grease are applied on both sides of the membrane. The mechanical influence of narrow strips and electrodes is assumed to be neglectable. After strips of aluminum tape are attached to the plastic frame and electrically connected to the 1/25/2015 
electrodes the sample is released from the stretching device and transferred to the testing setup, connected to the high voltage generator and restored to the prior defined dimension. In this prestretched state (Fig. 2-3), the membrane has a state of stretches $\left\{\lambda_{1}, \lambda_{2 p}\right\}$, and is of length $\lambda_{1 p} L_{1}(=10 \mathrm{~mm})$ and width $\lambda_{2 p} L_{2}(=100 \mathrm{~mm})$. In a current state (Fig. 2-4), the prestretched sample is connected to the voltage source, and has a state of stretches $\left\{\lambda_{1}, \lambda_{2}\right\}$.

The membranes are subject to all horizontal and vertical prestretch combinations in the range of $\lambda_{1 p}=1-7$, and $\lambda_{2 p}=1-7$ respectively. Voltage ramp rates of $1 \mathrm{~V} / \mathrm{s}, 5 \mathrm{~V} / \mathrm{s}, 50 \mathrm{~V} / \mathrm{s}$, and 100V/s are applied. We record a large set of voltage-stretch curves (Fig. 3). The horizontal prestretch $\lambda_{2 p}$ is indicated in each subfigure. The vertical prestretch $\lambda_{1 p}$ can be read by the intersection between the voltage-stretch curve and the $x$ coordinate, that is, the stretch at $=0$. The red, green, blue, or grey curve represents the experimental data for a voltage ramp rate of $1 \mathrm{~V} / \mathrm{s}, 5 \mathrm{~V} / \mathrm{s}, 50 \mathrm{OV} / \mathrm{s}$, or $100 \mathrm{~V} / \mathrm{s}$, respectively. Each curve terminates at a failure of electric breakdown, such that the electric current across the elastomer exceeds the current limit of the HV generator of $1 \mathrm{~mA}$. Since dielectric failure is also a phenomenon statistically distributed and can depend on sample preparation as well as on defects in the dielectric, measurements are repeated for each set of conditions. To allow a visual inspection of voltage induced deformation and breakdown phenomena the front plane view is recorded by a video camera.

For a membrane of any fixed prestretches $\left\{\lambda_{1 p}, \lambda_{2_{p}}\right\}$, the voltage-stretch curves obviously depend on the voltage ramp rates (Fig. 3). For example, for a membrane of prestretches $\{2,7\}$, as shown in the last subfigure in Fig. 3, the slope of the voltage-stretch curve increases when the voltage ramp rate increases. The observed rate effect is due to the viscoelasticity. As the voltage ramp rate increases, the membrane of a dielectric elastomer has less time for relaxation. In addition, the clamped membrane achieves giant voltage-induced deformation under certain conditions. For example, for a membrane of prestretches $\{2,6\}$, the 
stretch $\lambda_{1} / \lambda_{1 p}$ at failure attains 4.2 at $\quad=3099 \mathrm{~V}$ for $1 \mathrm{Vs}$, and attains 3.36 at $=4180 \mathrm{~V}$ for $100 \mathrm{~V} / \mathrm{s}$.

We observe three types of electromechanical phase transition (Videos 1, 2 and 3). As discussed in the last section, type I transition exhibits a discontinuous FW transition, and type II transition exhibits a continuous FW transition. Besides these FW transitions, we observe a type III transition as follows, which we term wrinkle-to-wrinkle (WW) transition. The flat membrane first forms wrinkles throughout the surface at a critical voltage. When the voltage increases to another critical value, the wavelength of the wrinkles suddenly reduces, accompanied by a jump in the deformation. These three types of transition will be interpreted in the following sections, using a model of viscoelasticity.

\section{A viscoelastic model}

In this section, we employ a model of viscoelasticity to analyze the experimental observations in Section 2. Aspects of the theory essential to the current work are briefly summarized here. For more details on the theory of viscoelastic dielectrics see Hong (2011), Zhao et al. (2011), and Foo et al. (2012). For more details on the nonlinear field theory on dielectric elastomers see Dorfmann and Ogden (2005), McMeeking and Landis (2005), Goulbourne et al. (2005), and Suo et al. (2008). For a review of theory of dielectric elastomers see Suo (2010).

In the reference state, the membraneis of length $L_{1}$, width $L_{2}$, and thickness $H$ (Fig. 1a). In the current state, the membrane is subject to voltage $\Phi$ through the thickness and forces $P_{1}$ and $P_{2}$ in the plane. Consequently, the membrane is of length $l_{1}$, width $l_{2}$, and thickness $h$, and gains charges $\pm Q$ on the two electrodes (Fig. 1b). Define the stretches by $\lambda_{1}=l_{1} / L_{1}, \lambda_{2}=l_{2} / L_{2}$ and $\lambda_{3}=h / H$, the stresses by $\sigma_{1}=P_{1} /\left(l_{2} h\right)$ and $\sigma_{2}=P_{2} /\left(l_{1} h\right)$, the electric field by $E=\Phi / h$, 
and the electric displacement by $D=Q /\left(l_{1} l_{2}\right)$. The elastomer is taken to be incompressible, so that

$$
\lambda_{1} \lambda_{2} \lambda_{3}=1
$$

This assumption is reasonable for elastomers, since the shape change of elastomers typically is much more significant than their volume change, especially when elastomers undergo large deformation.

We assume that the electric displacement is linear in the electric field:

$$
D=\varepsilon E
$$

where $\varepsilon$ is the permittivity of the dielectric elastomer. This assumption is based on the molecular picture as follows. The dielectric elastomer is a crosslinked network of flexible polymers. When the degree of crosslink is not large and the deformation is well below the extension limit, the polymer molecules can polarize freely, and dielectric behavior of the dielectric elastomer is similar to that of a polymer melt, independent of the deformation. This idealization is known as the model of ideal dielectric elastomer (Zhao et al., 2007), and is consistent with the experiments which showed that the permittivity of the dielectric elastomer (VHB) decreases negligibly (less than 5\%) when its area increases by 25 times (Kofod et al., 2003). Based on this assumption, the Helmholtz free energy density of the dielectric elastomer may consist of two parts,

$$
W\left(\lambda_{1}, \lambda_{2}, D, \xi_{1}, \xi_{2}, \ldots\right)=W_{\text {stretch }}\left(\lambda_{1}, \lambda_{2}, \xi_{1}, \xi_{2}, \ldots\right)+\frac{D^{2}}{2 \varepsilon}
$$

where $W_{\text {stretch }}$ is the Helmholtz free energy density associated with the stretching of the elastomer, $\xi_{1}, \xi_{2}, \ldots$ represent a set of internal variables that characterize the progress of viscoelastic and dielectric relaxation in the dielectric elastomer, and $D^{2} /(2 \varepsilon)$ is the Helmholtz free energy density associated with the polarization of the elastomer.

The mechanical and electrostatic equilibrium gives 


$$
\begin{gathered}
\sigma_{1}+\varepsilon E^{2}=\lambda_{1} \frac{\partial W_{\text {stretch }}\left(\lambda_{1}, \lambda_{2}, \xi_{1}, \xi_{2}, \ldots\right)}{\partial \lambda_{1}}, \\
\sigma_{2}+\varepsilon E^{2}=\lambda_{2} \frac{\partial W_{\text {stretch }}\left(\lambda_{1}, \lambda_{2}, \xi_{1}, \xi_{2}, \ldots\right)}{\partial \lambda_{2}} .
\end{gathered}
$$

Once the function of the free energy density $W_{\text {stretch }}\left(\lambda_{1}, \lambda_{2}, \xi_{1}, \xi_{2}, \ldots\right)$ is prescribed for the elastomer, (4) and (5) constitute the equations of state. However, the membrane is not in equilibrium with respect to viscoelastic and dielectric relaxation, so that

$$
\sum_{i} \frac{\partial W_{\text {stretch }}\left(\lambda_{1}, \lambda_{2}, \xi_{1}, \xi_{2}, \ldots\right)}{\partial \xi_{i}} \delta \xi_{i} \leq 0 .
$$

See Appendix $A$ for the derivation of (4-6).

In response to an electromechanical load, an elastomer may evolve toward a new state of equilibrium by various dissipative processes. Viscoelastic relaxation may be caused by slipping and sliding among polymer chains and rotation of joints among monomers. Dielectric relaxation may be induced by distortion of electron clouds and rotation of polar groups. Experiments have shown that for VHB, the viscoelastic relaxation time is several hundred seconds (Carpi et al., 2008; Keplinger et al., 2008; Zhao et al., 2011). Based on the experiments of dielectric polymers (Seki and Sato 1995; Reffaee et al, 2009), the dielectric relaxation time for dielectric elastomers, at room temperature, is assumed to be on the order of $10^{-6}$ seconds. Since our experiments are conducted within a time scale of minutes, we will ignore dielectric relaxation, but focus on viscoelastic relaxation.

We adopt a nonlinear rheological model to simulate the effect of viscoelasticity. The model consists of two parallel units - spring $\alpha$ in parallel with spring $\beta$ and a dashpot (Fig. 4). These two parallel units have the same net stretches. Spring $\alpha$ has stretches $\lambda_{1}$ and $\lambda_{2}$, which are exactly the same as those of the elastomer. Let us assume the stretches of spring $\beta$ are $\lambda_{1}^{e}$ and $\lambda_{2}^{e}$, and those of the dashpot are $\xi_{1}$ and $\xi_{2}$. We have 


$$
\lambda_{1}=\lambda_{1}^{e} \xi_{1}, \lambda_{2}=\lambda_{2}^{e} \xi_{2}
$$

For a dielectric elastomer, each polymer chain may have a finite length, known as the extension limit (Arruda and Boyce, 1993). When the polymer chains approach the stretch limit, the elastomer may stiffen sharply. Taking into account the strain-stiffening effect, we adopt the Gent (1996) model for both springs $\alpha$ and $\beta$

$$
\begin{aligned}
& W_{\text {stretch }}\left(\lambda_{1}, \lambda_{2}, \xi_{1}, \xi_{2}\right)=-\frac{\mu^{\alpha} J_{\lim }^{\alpha}}{2} \log \left(1-\frac{\lambda_{1}^{2}+\lambda_{2}^{2}+\lambda_{1}^{-2} \lambda_{2}^{-2}-3}{J_{\lim }^{\alpha}}\right), \\
& -\frac{\mu^{\beta} J_{\lim }^{\beta}}{2} \log \left(1-\frac{\lambda_{1}^{2} \xi_{1}^{-2}+\lambda_{2}^{2} \xi_{2}^{-2}+\lambda_{1}^{-2} \lambda_{2}^{-2} \xi_{1}^{2} \xi_{2}^{2}-3}{J_{\lim }^{\beta}}\right)
\end{aligned}
$$

where $\mu^{\alpha}$ and $\mu^{\beta}$ represent the small-strain shear modulus of spring $\alpha$ and $\beta$, respectively, and $J_{\lim }^{\alpha}$ and $J_{\text {lim }}^{\beta}$ are the material constants related to the extension limits of spring $\alpha$ and $\beta$, respectively. Substituting (8) into (4) and (5), we have

$$
\begin{aligned}
& \sigma_{1}+\varepsilon E^{2}=\frac{\mu^{\alpha}\left(\lambda_{1}^{2}-\lambda_{1}^{-2} \lambda_{2}^{-2}\right)}{1-\left(\lambda_{1}^{2}+\lambda_{2}^{2}+\lambda_{1}^{-2} \lambda_{2}^{-2}-3\right) / J_{\lim }^{\alpha}}+\frac{\mu^{\beta}\left(\lambda_{1}^{2} \xi_{1}^{-2}-\xi_{1}^{2} \xi_{2}^{2} \lambda_{1}^{-2} \lambda_{2}^{-2}\right)}{1-\left(\lambda_{1}^{2} \xi_{1}^{-2}+\lambda_{2}^{2} \xi_{2}^{-2}+\xi_{1}^{2} \xi_{2}^{2} \lambda_{1}^{-2} \lambda_{2}^{-2}-3\right) / J_{\lim }^{\beta}} \\
& \sigma_{2}+\varepsilon E^{2}=\frac{\mu^{\alpha}\left(\lambda_{2}^{2}-\lambda_{1}^{-2} \lambda_{2}^{-2}\right)}{1-\left(\lambda_{1}^{2}+\lambda_{2}^{2}+\lambda_{1}^{-2} \lambda_{2}^{-2}-3\right) / J_{\lim }^{\alpha}}+\frac{\mu^{\beta}\left(\lambda_{2}^{2} \xi_{2}^{-2}-\xi_{1}^{2} \xi_{2}^{2} \lambda_{1}^{-2} \lambda_{2}^{-2}\right)}{1-\left(\lambda_{1}^{2} \xi_{1}^{-2}+\lambda_{2}^{2} \xi_{2}^{-2}+\xi_{1}^{2} \xi_{2}^{2} \lambda_{1}^{-2} \lambda_{2}^{-2}-3\right) / J_{\lim }^{\beta}}
\end{aligned}
$$

In the rheological model as shown in Fig. 4, the dashpot undergoes the same stresses as spring $\beta$ does. The strain rates in the dashpot are $\xi_{1}^{-1} d \xi_{1} / d t$ and $\xi_{2}^{-1} d \xi_{2} / d t$, respectively. Assuming the dashpot a Newtonian fluid with viscosity $\eta$, we have

$$
\begin{aligned}
& \frac{d \xi_{1}}{\xi_{1} d t}=\frac{1}{3 \eta}\left(\frac{\mu^{\beta}\left(\lambda_{1}^{2} \xi_{1}^{-2}-\xi_{1}^{2} \xi_{2}^{2} \lambda_{1}^{-2} \lambda_{2}^{-2}\right)}{1-\left(\lambda_{1}^{2} \xi_{1}^{-2}+\lambda_{2}^{2} \xi_{2}^{-2}+\xi_{1}^{2} \xi_{2}^{2} \lambda_{1}^{-2} \lambda_{2}^{-2}-3\right) / J_{\lim }^{\beta}}-\frac{\mu^{\beta}\left(\lambda_{2}^{2} \xi_{2}^{-2}-\xi_{1}^{2} \xi_{2}^{2} \lambda_{1}^{-2} \lambda_{2}^{-2}\right) / 2}{1-\left(\lambda_{1}^{2} \xi_{1}^{-2}+\lambda_{2}^{2} \xi_{2}^{-2}+\xi_{1}^{2} \xi_{2}^{2} \lambda_{1}^{-2} \lambda_{2}^{-2}-3\right) / J_{\lim }^{\beta}}\right) \\
& \frac{d \xi_{2}}{\xi_{2} d t}=\frac{1}{3 \eta}\left(\frac{\mu^{\beta}\left(\lambda_{2}^{2} \xi_{2}^{-2}-\xi_{1}^{2} \xi_{2}^{2} \lambda_{1}^{-2} \lambda_{2}^{-2}\right)}{1-\left(\lambda_{1}^{2} \xi_{1}^{-2}+\lambda_{2}^{2} \xi_{2}^{-2}+\xi_{1}^{2} \xi_{2}^{2} \lambda_{1}^{-2} \lambda_{2}^{-2}-3\right) / J_{\lim }^{\beta}}-\frac{\mu^{\beta}\left(\lambda_{1}^{2} \xi_{1}^{-2}-\xi_{1}^{2} \xi_{2}^{2} \lambda_{1}^{-2} \lambda_{2}^{-2}\right) / 2}{1-\left(\lambda_{1}^{2} \xi_{1}^{-2}+\lambda_{2}^{2} \xi_{2}^{-2}+\xi_{1}^{2} \xi_{2}^{2} \lambda_{1}^{-2} \lambda_{2}^{-2}-3\right) / J_{\lim }^{\beta}}\right)
\end{aligned}
$$


With $\eta>0$, this model can satisfy the thermodynamic inequality (6). Once the mechanical and electrical loading functions, $P_{1}(t), P_{2}(t)$ and $\Phi(t)$, are prescribed, we can use Eqs. (9-12) for the evolution of $\lambda_{1}(t), \lambda_{2}(t), \xi_{1}(t)$ and $\xi_{2}(t)$.

We then fit this viscoelastic model to the experimental data. Fig. 5 plots the voltagestretch curves for the membrane of prestretches $\{2,7\}$ at voltage ramp rates of $1 \mathrm{~V} / \mathrm{s}, 5 \mathrm{~V} / \mathrm{s}$ and 50 V/s. The solid curves represent the experimental data, while the dashed curves represent theoretical calculations. The material parameters used in the calculations are $\mu^{\alpha}=20 \mathrm{kPa}, \mu^{\beta}=$ 20okPa, $J_{\text {lim }}^{\alpha}=155, J_{\text {lim }}^{\beta}=155, \eta / \mu^{\beta}=50$, and $\varepsilon=4.12 \times 10^{-11} \mathrm{~F} / \mathrm{m}$. The open circles and diamonds represent that the membranes suffer loss of tension and form wrinkles in theoretical predictions and experimental observations, respectively.

We calculate the dashed curves, taking the following procedures. First, we assume that at $\Phi=0$, the dashpot is fully relaxed. That is, the initial conditions are as follows: $\lambda_{1}=\lambda_{1 p}$, $\lambda_{2}=\lambda_{2 p}, \xi_{1}=\lambda_{1 p}$, and $\xi_{2}=\lambda_{2 p}$. The load $P_{1}$ in the vertical direction can be determined by (9)

$$
\frac{P_{1}}{L_{2} H}=\frac{\mu^{\alpha}\left(\lambda_{1 p}-\lambda_{1 p}^{-3} \lambda_{2 p}^{-2}\right)}{1-\left(\lambda_{1 p}^{2}+\lambda_{2 p}^{2}+\lambda_{1 p}^{-2} \lambda_{2 p}^{-2}-3\right) / J_{\lim }^{\alpha}}
$$

We increase voltage at a constant ramp rate $K$,

$$
\Phi=K t
$$

where $t$ is time. When the voltage is small, the clamps hold the horizontal stretch fixed, so that $\lambda_{2}$ is a constant $\left(=\lambda_{2 p}\right)$. We use Eqs (9) and (11-12) for the evolution of $\lambda_{1}(t), \xi_{1}(t)$ and $\xi_{2}(t)$. However, the experimental observations of wrinkles, as represented by diamonds in Fig. 5, indicate that the clamps do not always hold the horizontal stretch fixed. In the theoretical calculation, the horizontal tensile stress can be determined by Eq (10). The calculation confirms 
that, as the voltage ramps up, the horizontal tensile stress decreases and eventually vanishes at a specific state of voltage and stretch, marked by an open circle in the dashed curve.

After the membrane forms wrinkles, we set $\sigma_{2}=0$,

$$
P_{2}=\mathrm{O}
$$

That is, after loss of tension, the loading conditions are described by Eqs (13-15). We record the time at loss of tension $t_{0}$, and we have the initial conditions as $\lambda_{1}=\lambda_{1}\left(t_{0}\right), \lambda_{2}=\lambda_{2 p}, \xi_{1}=\xi_{1}\left(t_{0}\right)$, and $\xi_{2}=\xi_{2}\left(t_{0}\right)$. Then we will use Eqs. (9-12) for the evolution of $\lambda_{1}(t), \lambda_{2}(t), \xi_{1}(t)$ and $\xi_{2}(t)$ after the membrane forms wrinkles.

Video 2 shows the sequence of the states of a membrane of prestretches $\{2,7\}$ as the voltage increases at a ramp rate of $50 \mathrm{~V} / \mathrm{s}$. The flat membrane forms wrinkles throughout the surfaces simultaneously at $\quad=2843 \mathrm{~V}$. This FW transition (type II transition) is accompanied by a continuous change in the vertical displacement, as shown in Fig.5.

In the following section, we will study a discontinuous FW transition (type I transition), by using a model of viscoelasticity. The discontinuous FW transition is associated with snapthrough instability.

\section{Discontinuous flat-to-wrinkle transition}

Video 1 shows a discontinuous FW transition in a membrane of prestretches $\{2,4\}$ at a voltage ramp rate of $5 \mathrm{~V} / \mathrm{s}$. The membrane is flat when the voltage is small. When the voltage reaches a critical value, wrinkles may nucleate in small regions of a flat membrane, and then the wrinkled regions grow at the expense of the flat regions. This transition is accompanied by a sudden snap in the vertical displacement.

This transition can be interpreted by using an elastic model of a dielectric elastomer (Zhu et al., 2012). As illustrated in Fig. 6a, a clamped membrane of a dielectric elastomer may 
have an $\mathrm{N}$-shaped (going up, down and up again) voltage-charge curve. When the charge is small, the voltage needed to maintain the charge increases with the charge. The membrane is flat, since it is subject to a horizontal tensile stress from the clamps. As the charge increases, the membrane expands in area. However, the membrane is constrained by the clamps in the horizontal direction (see Fig. 2), so that the horizontal tensile stress reduces. At a certain state of voltage and charge, marked by an open circle in Fig. 6a, the horizontal stress vanishes, and the membrane forms wrinkles. After forming wrinkles, the membrane thins down appreciably, and the voltage needed to maintain the charge decreases. As a result, the voltage reaches a peak in the voltage-charge diagram. Upon approaching the extension limit of the polymer, the voltagecharge curve goes up again due to the strain-stiffening effect. For the membrane with an Nshaped voltage-charge curve, flat and wrinkled regions can coexist at the voltage of transition

*. Analogous to the Maxwell rule in thermodynamic phase transition, the voltage for coexistent states is at the level such that two shaded regions have the equal area, and the flat state $A$ and the wrinkled state $B$ have the same energy density. When voltage reaches $\quad$ *, the membrane may exhibit a discontinuous transition from the flat state to the wrinkled state, accompanied by a sudden jump in the charge or the deformation.

In this paper, we attempt to analyze the effect of viscoelasticity. Using a model of viscoelasticity, it is impossible to plot the full curve - going up, down and up again (Fig. 6a), since it is hard to evaluate the time dependent deformation especially after the voltage reaches the peak. We propose to analyze the discontinuous FW transition (type I transition) and snap through instability in a viscoelastic dielectric elastomer, by taking the following steps.

Step 1: analyze the evolution of $\lambda_{1}(t), \xi_{1}(t)$, and $\xi_{2}(t)$ when the membrane is flat. This step is similar to what we conduct last section to analyze the continuous FW transition (type II transition). The loading conditions are given by Eqs (13-14), the initial conditions are $\lambda_{1}=\lambda_{1 p}$, $\xi_{1}=\lambda_{1 p}$, and $\xi_{2}=\lambda_{2 p}$, and Eqs (9) and (11-12) are employed to calculate $\lambda_{1}(t), \xi_{1}(t)$, and $\xi_{2}(t)$. 
Eq (10) is then used to calculate the horizontal tensile stress. At time $t_{0}$, the membrane suffers loss of tension, as marked by the open circle in Fig. 6b. At any time $t \leq t_{0}$, the stretches of the elastomer are $\lambda_{1}^{F}(t)$ and $\lambda_{2}^{F}(t)\left(=\lambda_{2 p}\right)$, respectively, and the stretches of the dashpot are $\xi_{1}^{F}(t)$ and $\xi_{2}^{F}(t)$, respectively. The superscript $F$ denotes the membrane is at the flat state.

Step 2: examine, at any time $t\left(\leq t_{0}\right)$, whether a wrinkled state exists or not, which can satisfy the equations of state - Eqs (9-10). It should be noted that before loss of tension, the membrane is clamped, $\lambda_{2}$ is fixed $\left(=\lambda_{2 p}\right)$, while $\sigma_{2}$ decreases with time. However, after loss of tension, the membrane is in effect unclamped, $\sigma_{2}$ vanishes, but $\lambda_{2}$ becomes to be a variable. For this wrinkled state, we assume that the stretches of the elastomer are $\lambda_{1}^{W}(t)$ and $\lambda_{2}^{W}(t)$, and the stretches of the dashpot are $\xi_{1}^{W}(t)$ and $\xi_{2}^{W}(t)$. The superscript $W$ denotes the membrane is at the wrinkled state. We assume that at anytime $t$, the membrane may snap from one state of equilibrium to another, due to a disturbance, which may be large enough to overcome the energy barrier. The snap may occur rapidly, as confirmed by the experiments (see Videos 1). During snap through instability, we may ignore the deformation change of the dashpot, and assume that the membrane is elastic. With $\xi_{1}^{F}=\xi_{1}^{W}$, and $\xi_{2}^{F}=\xi_{2}^{W}$, at any time $t\left(\leq t_{0}\right)$, we can solve Eqs (910) for the two unknowns $\lambda_{1}^{W}$ and $\lambda_{2}^{W}$. The calculations show that when the voltage is less than a certain value (say, the voltage for State $A$ in Fig. 6b), we can not get a solution for the wrinkled state, and the flat state is the only state of equilibrium. However, when the voltage is more than this critical value, both the flat and wrinkled states can be the states of equilibrium, such as States $A$ and $D, B$ and $E$, or $C$ and $F$, as illustrated in Fig. 6 .

Step 3: Compare the free energy densities of the system associated with the flat and wrinkled states. The electromechanical phase transition may occur at 


$$
W_{\text {stretch }}\left(\lambda_{1}^{F}, \xi_{1}^{F}, \xi_{2}^{F}\right)-\left(\frac{P_{1}}{L_{2} H}\right) \lambda_{1}^{F}-\frac{\varepsilon}{2}\left(\frac{\Phi}{H}\right)^{2}\left(\lambda_{1}^{F} \lambda_{2}^{F}\right)^{2}=W_{\text {stretch }}\left(\lambda_{1}^{W}, \lambda_{2}^{W}, \xi_{1}^{W}, \xi_{2}^{W}\right)-\left(\frac{P_{1}}{L_{2} H}\right) \lambda_{1}^{W}-\frac{\varepsilon}{2}\left(\frac{\Phi}{H}\right)^{2}\left(\lambda_{1}^{W} \lambda_{2}^{W}\right)^{2}
$$

See Appendix $B$ for the derivation of the condition for electromechanical phase transition in a viscoelastic dielectric elastomer. As illustrated in Fig. 6b, States $B$ and $E$ may have the same energy density at $=*$. The calculations show that when $<{ }^{*}$, the free energy density associated with the flat state is less than that associated with the wrinkled state, and the flat state is more stable. On the other hand, when $>{ }^{*}$, the energy density associated with the flat state is more than that associated with the wrinkled state, and the wrinkled state is more stable. This conclusion is consistent with that for an elastic material (Huang and Suo, 2011; Zhu et al, 2012).

Step 4: calculate the voltage-charge/stretch curve after electromechanical phase transition. First we can record the time for phase transition $t_{1}\left(<t_{0}\right)$. Then we can use Eqs (9-12) for the evolution of $\lambda_{1}(t), \lambda_{2}(t), \xi_{1}(t)$, and $\xi_{2}(t)$, based on the initial conditions: $\lambda_{1}=\lambda_{1}^{W}\left(t_{1}\right)$, $\lambda_{2}=\lambda_{2}^{W}\left(t_{1}\right), \xi_{1}=\xi_{1}^{W}\left(t_{1}\right)$, and $\xi_{2}=\xi_{2}^{W}\left(t_{1}\right)$. Note that $\xi_{1}^{F}\left(t_{1}\right)=\xi_{1}^{W}\left(t_{1}\right)$, and $\xi_{2}^{F}\left(t_{1}\right)=\xi_{2}^{W}\left(t_{1}\right)$.

Fig. 7 illustrates the use of the model of viscoelasticity for simulation of the membrane of prestretches $\{2,4\}$. The mechanical load in the vertical direction is constant (Fig. 7a). The membrane is then subject to voltage with a ramp rate of $5 \mathrm{~V} / \mathrm{s}$ (Fig. $7 \mathrm{~b}$ ). When the voltage is small, the membrane is clamped (with $\lambda_{2 p}=4$ ) and flat. Using Eqs (9) and (11-12), we calculate the evolution of $\lambda_{1}(t), \xi_{1}(t)$, and $\xi_{2}(t)$. Using Eq (10), we calculate the evolution of the horizontal stress $\sigma_{2}(t)$ (Fig. 7c). At $t=613 \mathrm{~s}$, the horizontal stress vanishes, and the membrane forms wrinkles. The loss of tension is represented by an open circle in Figs 7c-7e. The evolution of $\xi_{1}(t)$ and $\xi_{2}(t)$ is shown in Fig. 7d. As discussed above, we always examine whether another wrinkled state of equilibrium exists, to which the flat state may snap. Fig. 7e shows the evolution 
of $\lambda_{1}(t)$ and $\lambda_{2}(t)$. When $t \geq 568 \mathrm{~s}$, another wrinkled state of equilibrium may exist. Fig. $7 \mathrm{f}$ compares the energy densities associated with the flat and wrinkled states. When $t=582 \mathrm{~s}$, the flat and wrinkled states have the same energy density. Fig. $7 \mathrm{~g}$ shows the evolution of the stretches of the elastomer. The transition from the flat state to the wrinkled state occurs at $t=$ 582s. Fig. 7h plots the voltage-stretch curves. The solid curve represents the experimental data. The dashed curve represents the theoretical calculations. The theory predicts that when the voltage reaches a critical value, the membrane may transform from a flat state to a wrinkled state, accompanied with a sudden snap in the vertical displacement. The theoretical predictions are qualitatively consistent with the experimental observations.

\section{Wrinkle-to-wrinkle transition}

On carefully examining the experimental videos of the membranes of different prestretches, subject to voltage with different ramp rates (Fig. 3), we find a WW transition. When the voltage reaches a critical value, the flat membrane transforms to the wrinkled membrane continuously, similar to a continuous FW transition (type II transition). However, when the voltage increases to another critical value, the wavelength of the wrinkles suddenly reduces, accompanied by a jump in the vertical displacement.

We can interpret the WW transition by using an elastic model (Fig. 8a). When the charge is small, the voltage increases with the charge. At a critical voltage, the membrane suffers loss of tension and forms wrinkles, as represented by an open circle (Fig.8a). After loss of tension, the voltage-charge curve still goes up, and then down, and finally up again. At another critical voltage *, which makes the areas of the shaded regions same, the membrane transforms from the wrinkled state with a large wavelength to that with a small wavelength. Using a viscoelastic model for dielectric elastomers, * can be determined by equating the energy densities of wrinkled states 1 and 2 (Fig. 8b). 
Fig. 9 illustrates the evolution and snap in the membrane of pre-stretches $\{6,2\}$ at a voltage ramp rate of $50 \mathrm{~V} / \mathrm{s}$. The membrane is subject to a constant mechanical load (Fig. 9a), and then is subject to a ramping voltage (Fig. 9b). As shown in Fig. 9c, the horizontal tensile stress reduces as the voltage increases. At $t=43.4 \mathrm{~s}$, the horizontal tensile stress vanishes. The horizontal stress is set to zero after the membrane forms wrinkles. Fig. 9d shows the evolution of the dashpot deformation, which increases continuously. Fig. 9e shows the evolution of the elastomer deformation. When $t<58 \mathrm{~s}$, there is only one state of equilibrium. When $t \geq 58 \mathrm{~s}$, another wrinkled state of equilibrium with smaller wavelength exists. Fig. 9 f compares the free energy densities associated with the two wrinkled states. When $t=58.3 \mathrm{~s}$, the two wrinkled states have the same energy densities. Fig. $9 g$ shows the evolution of the elastomer deformation.

Fig. 10a plots the voltage-stretch curves of the membrane of prestretches $\{6,2\}$ at a voltage ramp rate of $50 \mathrm{O} / \mathrm{s}$. The solid curve represents the experimental data, while the dashed curve represents the theoretical predictions. The open circle and diamond in the theoretical and experimental curves represent that the membrane suffers loss of tension and forms wrinkles. The membrane then transforms from the wrinkled state with a large wavelength to that with a small wavelength suddenly, and suffers a jump in the vertical displacement. Figs. 10b-10g, as well as Video 3, show the sequence of the states of the membrane of prestretches $\{6,2\}$. The membrane is flat in the absence of voltage (Fig. 10b). When the voltage is small, the membrane remains at the flat state (Fig. 10c). The entire flat membrane forms wrinkles throughout the membrane at $\Phi=2850 \mathrm{O}$. (Fig. 10d). Then the wavelength of the wrinkles decreases dramatically within a time of about $3 \mathrm{~s}$, during which the voltage increases about $150 \mathrm{~V}$ (Figs. 10e-10f). Meanwhile, the vertical stretch $\lambda_{1}$ suddenly increases from 7 to 8 (see Fig. 10a). Finally, the membrane suffers a failure of dielectric breakdown (Fig. 10g) at about $3158 \mathrm{~V}$.

\section{Effects of viscoelasticity on electromechanical behavior}


Dielectric breakdown occurs when an electric field exceeds a certain limit, which first induces a nonlinear increase of current flow over time, then results in a conductive path throughout the membrane. For a dielectric elastomer, typically, the voltage for dielectric breakdown is a monotonically decreasing function of the stretch (Kofod et al., 2003; Plante and Dubowsky, 2006; Huang et al., 2012). This phenomenon can be interpreted as follows. As the in-plane stretches of the elastomer increase, the thickness of the elastomer decreases, and the voltage for dielectric breakdown decreases. It should be noted that, independent from experimental conditions, the measured electric field/voltage for breakdown is statistically distributed since dielectric breakdown might occur first at weak points in the material such as impurities, flaws, or cracks. Weak points might also be induced in the process of sample preparation.

In the schematic voltage-stretch diagrams in Fig. 11, the black curve represents the states of equilibrium for a clamped membrane of a dielectric elastomer, while the red curve represents the condition of dielectric breakdown. The open circle represents that the membrane suffers loss of tension and forms wrinkles, and the two solid circles represent the two states that have the same energy density and can coexist. We identify three types of transition, according to the black curve which may combine loss of tension and snap through instability. According to where the red and black curves intersect, we indentify nine kinds of representative behavior as follows. For type I-a behavior, the membrane suffers electrical breakdown prior to discontinuous FW transition. For type I-b behavior, the flat state is below the breakdown condition, while the wrinkled state suffers breakdown. As a result, the discontinuous FW transition is not observable, and the membrane fails at a flat state. For type I-c behavior, both the flat state and the wrinkled state are below the breakdown condition, and the discontinuous FW transition is observable (Video 1). For type II-a behavior, the membrane suffers electrical breakdown prior to continuous FW transition. For type II-b behavior, a continuous FW transition is observable (Video 2), since the wrinkled state is below the breakdown condition. 
For type III-a behavior, the membrane suffers electrical breakdown prior to loss of tension. For type III-b behavior, the membrane suffers electrical breakdown after loss of tension, but before snap through instability. For type III-c behavior, the wrinkled state with a large wavelength is below the dielectric breakdown condition, while that with a small wavelength suffers breakdown. The discontinuous WW transition is not observable. The continuous FW transition is observable, and the membrane fails at a wrinkled state. For type III-d behavior, both the wrinkled state with a large wavelength and that with a small wavelength are below the dielectric breakdown condition. As a result, both continuous FW transition and discontinuous WW transition are observable (Video 3).

The clamped membrane of a dielectric elastomer shows different kinds of electromechanical behavior, dependent on prestretches and voltage ramp rates. Fig. 12 summarizes the electromechanical behavior of the membrane mapped on the plane of $\left(\lambda_{1 p}, \lambda_{2 p}\right)$, at different voltage ramp rates. The theory predicts that the voltage-stretch curve exhibits a type I transition in the grey area, a type II transition in the green area, and a type III transition in the blue area, respectively. The diamond (in grey), triangle (in green), and circle (in blue) corresponds to the experimental observations for a membrane of specific prestretches $\left(\lambda_{1 p}, \lambda_{2 p}\right)$. The color of these symbols corresponds to the observed behavior of electromechanical transition. The grey solid diamond represents a type I-c behavior - a discontinuous FW transition (Video 1), and the grey open diamond represents a type I-a/b behavior. For both type I-a and type I-b, the membrane may fail at a flat state, and it is difficult to distinguish them. The green solid triangle represents a type II-b behavior - a continuous FW transition (Video 2). In the experiments, type II-b behavior is more frequently observed than type II-a behavior, for a membrane of large prestretches. The blue solid circle represents a type III-d behavior, which consists of a continuous FW transition and a discontinuous WW transition (Video 3). The blue open circle represents a type III-b/c behavior. For both type III-b and type III-c, the membrane may 
transform from a flat state to a wrinkled state continuously, and then fail at a wrinkled state. It is difficult to distinguish type III-b/c behavior experimentally. The type III-a behavior is seldom observed in the experiments.

The experimental observations, related to the behavior of type I-a, II-a, or III-a, may appear to be similar. That is, the membrane fails at a flat state, which might be due to a low dielectric strength of specimen under test. In addition, the experimental observations, related to the behavior of type II-b and III-b, are also similar to each other. That is, the membrane may transform from a flat state to a wrinkled state continuously, and then fail at a wrinkled state. However, they correspond to different prestretches, and can be identified based on theoretical analyses of voltage-charge/stretch curves.

Prestretches of both $\lambda_{1 p}$ and $\lambda_{2 p}$ may affect electromechanical behavior of the membrane of a dielectric elastomer. In each subfigure in Fig. 12, the voltage ramp rate is fixed. As we can see, when $\lambda_{1 p}$ and $\lambda_{2 p}$ are large, the membrane may avert snap through instability type II transition. With a large $\lambda_{1 p}$ but a small $\lambda_{2 p}$, the voltage-stretch curve of the membrane exhibits a type III transition. With a large $\lambda_{2 p}$ but a small $\lambda_{1 p}$, the voltage-stretch curve exhibits type I transition. It should be noted for both type I and type III transitions, the membrane undergoes snap through instability. In the type III transition, the membrane suffers snap through instability after loss of tension. In the type I transition, the membrane suffers snap through instability before loss of tension.

The theoretical predictions are based on independently measured parameters, yet it agrees well with experimental observations regarding the influence of viscoelasticity. An example is shown in Fig. 12, where the voltage ramp rate plays an important role to affect the electromechanical behavior of the membrane. For example, for $\lambda_{1 p}=4$ and $\lambda_{2 p}=4$, the voltage-stretch curve exhibits a type II transition when the loading rate is $1 \mathrm{~V} / \mathrm{s}$ or $5 \mathrm{~V} / \mathrm{s}$, but exhibits a type III transition when the loading rate is $50 \mathrm{v} / \mathrm{s}$ or $100 \mathrm{~V} / \mathrm{s}$. Videos 4 and 5 compare 
the electromechanical behavior of the membrane of $\{4,4\}$ at a voltage ramp rate of $5 \mathrm{~V} / \mathrm{s}$ and $100 \mathrm{~V} / \mathrm{s}$. As shown in Video e, the membrane can transform from the flat state to the wrinkled state continuously. As shown in Video 5, the membrane may form wrinkles first. Then the wrinkles having larger wavelength can transform to those of smaller wavelength suddenly, accompanied with a sudden displacement change.

In the current experiments, it is difficult to measure the dielectric breakdown field of the clamped membrane, because the horizontal stretch $\lambda_{2}$ becomes unknown when the membrane forms wrinkles. Kofod et al. (2003) measured dielectric strengths of a dielectric elastomer by fixing the membrane to rigid metals, and found that the dielectric strength may increase with the prestretches. Huang et al. (2012) employed another method to measure the dielectric strength. A thin sheet of elastomer is first prestretched, crossed-wire electrodes are then attached to the two surfaces of the elastomer, and finally both the elastomer and the electrodes are embedded in a stiff polymer. The dielectric strengths measured by Huang et al. (2012) are more than double those in Kofod et al. (2003). This dependence of the breakdown field on testing methods and materials lies at the boundaries of material science, nonlinear elasticity and thermodynamics. The phenomenon is not well understood, and deserves further investigations.

\section{Concluding remarks}

We investigate electromechanical behavior of a viscoelastic dielectric elastomer by measuring voltage-stretch curves under various prestretches and voltage rates. We find that a minimal analytic model based on physical mechanisms and independently determined material parameters is capable of describing the entire set of experimental observations. Besides continuous and discontinuous flat-to-wrinkle transitions, we discovered a new phenomenon, that is, a wrinkle-to-wrinkle transition. In that case, the entire flat membrane initially experiences a flat-to-wrinkle transition at a critical voltage. At a second critical voltage, the wavelength of the wrinkles suddenly decreases discontinuously, accompanied by a sudden jump 
in the voltage charge/stretch curve. The phase transitions occur widely in these experiments. Both the prestretches and the voltage ramp rate determine the global physical state of the dielectric elastomer. For example, a membrane of intermediate pre-stretches may suffer instability when the voltage ramp rate is high and avert it when the voltage ramp rate is low. The wrinkle-to-wrinkle transition and the corresponding snap through instability are also captured by the analytical model, which gives an insight in the temporal behavior of a viscoelastic dielectric elastomer. This study demonstrates that viscoelasticity can be properly included in a model, and that it is indeed essential to any attempt at modeling the complex temporal behavior of stable and unstable dielectric elastomers.

\section{Acknowledgements}

G.K. and M.K. thank the German Federal Ministry of Education and Research (BMBF), for support via grant No. 03X5511 "KompAkt" (WING-NanoFutur). Z.S. acknowledges the support of NSF MRSEC (DMR-o820484). J.Z. acknowledges support from Ministry of Education, Singapore (MOE, R -265-000-444-133).

\section{Appendix A. Nonequilibrium thermodynamics of a dielectric elastomer}

Fig. 1 illustrates a membrane of a dielectric elastomer. Thermodynamics requires that the increase in the Helmholtz free energy of the membrane $F$ should be not more than the total work done by the forces and the voltage. That is

$$
\delta F \leq P_{1} \delta l_{1}+P_{2} \delta l_{2}+\Phi \delta Q
$$

where $Q$ is the charges on the electrodes. We can think the membrane as a stretchable capacitor, so that

$$
Q=\varepsilon \frac{\Phi}{H} L_{1} L_{2}\left(\lambda_{1} \lambda_{2}\right)^{2}
$$


Define the density of the Helmholtz free energy of the membrane as $W=F /\left(L_{1} L_{2} H\right)$. Dividing (A1) by the volume of the membrane, $L_{1} L_{2} H$, we have

$$
\delta W \leq \frac{P_{1}}{L_{2} H} \delta \lambda_{1}+\frac{P_{2}}{L_{1} H} \delta \lambda_{2}+2 \varepsilon\left(\frac{\Phi}{H}\right)^{2}\left(\lambda_{1} \lambda_{2}^{2} \delta \lambda_{1}+\lambda_{1}^{2} \lambda_{2} \delta \lambda_{2}\right)
$$

Substituting (3) into (A3), we obtain

$$
\begin{gathered}
{\left[\frac{\delta W_{\text {stretch }}\left(\lambda_{1}, \lambda_{2}, \xi_{1}, \xi_{2}, \ldots\right)}{\delta \lambda_{1}}-\frac{P_{1}}{L_{2} H}-\varepsilon\left(\frac{\Phi}{H}\right)^{2} \lambda_{1} \lambda_{2}^{2}\right] \delta \lambda_{1}+\left[\frac{\delta W_{\text {stretch }}\left(\lambda_{1}, \lambda_{2}, \xi_{1}, \xi_{2}, \ldots\right)}{\delta \lambda_{2}}-\frac{P_{2}}{L_{1} H}-\varepsilon\left(\frac{\Phi}{H}\right)^{2} \lambda_{1}^{2} \lambda_{2}\right] \delta \lambda_{2}} \\
+\sum_{i} \frac{\delta W_{\text {stretch }}\left(\lambda_{1}, \lambda_{2}, \xi_{1}, \xi_{2}, \ldots\right)}{\delta \xi_{i}} \delta \xi_{i} \leq 0
\end{gathered}
$$

We assume that the membrane is in mechanical and electrostatic equilibrium, so that

$$
\begin{gathered}
\sigma_{1}+\varepsilon E^{2}=\lambda_{1} \frac{\partial W_{\text {stretch }}\left(\lambda_{1}, \lambda_{2}, \xi_{1}, \xi_{2}, \ldots\right)}{\partial \lambda_{1}} \\
\sigma_{2}+\varepsilon E^{2}=\lambda_{2} \frac{\partial W_{\text {stretch }}\left(\lambda_{1}, \lambda_{2}, \xi_{1}, \xi_{2}, \ldots\right)}{\partial \lambda_{2}}
\end{gathered}
$$

However, the membrane is not in equilibrium with respect to viscoelastic and dielectric relaxation, so that

$$
\sum_{i} \frac{\partial W_{\text {stretch }}\left(\lambda_{1}, \lambda_{2}, \xi_{1}, \xi_{2}, \ldots\right)}{\partial \xi_{i}} \delta \xi_{i} \leq 0
$$

\section{Appendix B. Conditions for discontinuous phase transition in a viscoelastic dielectric elastomer}

During a discontinuous phase transition, the flat and wrinkled regions can coexist, as illustrated in Fig. B1. In the reference state, the flat region is of length $L_{1}^{F}$, width $L_{2}$ and thickness $H$, while the wrinkled region is of length $L_{1}^{W}$, width $L_{2}$, and thickness $H$. We have

$$
L_{1}^{F}+L_{1}^{W}=L_{1}
$$


In the current state, for the flat region, the stretches of the elastomer are $\lambda_{1}^{F}$ and $\lambda_{2}^{F}\left(=\lambda_{2 p}\right)$, and the stretches of the dashpot are $\xi_{1}^{F}$ and $\xi_{2}^{F}$. For the wrinkled region, the stretches of the elastomer are $\lambda_{1}^{W}$ and $\lambda_{2}^{W}$, and the stretches of the dashpot are $\xi_{1}^{W}$ and $\xi_{2}^{W}$. We have

$$
L_{1}^{\prime} \lambda_{1}^{\prime}+L_{1}^{\prime \prime} \lambda_{1}^{\prime \prime}=l_{1}
$$

The free energy of the membrane in the current state consists of two parts - one for the flat region; the other for the wrinkled one. The free energy density of the flat region depends on three variables: $\lambda_{1}^{F}, \xi_{1}^{F}$ and $\xi_{2}^{F}$, while that of the wrinkled region depends on four variables: $\lambda_{1}^{W}, \lambda_{2}^{W}, \xi_{1}^{W}$ and $\xi_{2}^{W}$. Based on (3), we obtain the free energy of the membrane

$$
F=L_{2} H L_{1}^{F} W_{\text {stretch }}\left(\lambda_{1}^{F}, \xi_{1}^{F}, \xi_{2}^{F}\right)+L_{2} H L_{1}^{W} W_{\text {stretch }}\left(\lambda_{1}^{W}, \lambda_{2}^{W}, \xi_{1}^{W}, \xi_{2}^{W}\right)+\frac{\varepsilon}{2}\left(\frac{\Phi}{H}\right)^{2} L_{2} H\left[L_{1}^{F}\left(\lambda_{1}^{F} \lambda_{2 p}\right)^{2}+L_{1}^{W}\left(\lambda_{(\mathrm{B} 3)^{W}}^{W} \lambda^{W}\right)^{2}\right]
$$

Thermodynamics dictates that the increase in the free energy of the membrane should not be more than the work done by the force $P_{1}$ and voltage $\Phi$. We have

$$
\delta F \leq P_{1} \delta l_{1}+\Phi \delta Q
$$

where $Q$ is the charges on the electrodes

$$
Q=\varepsilon \Phi\left[\frac{L_{1}^{F} L_{2}}{H}\left(\lambda_{1}^{F} \lambda_{2 p}\right)^{2}+\frac{L_{1}^{W} L_{2}}{H}\left(\lambda_{1}^{W} \lambda_{2}^{W}\right)^{2}\right]
$$

The free energy (B3) is a function of eight independent variables: $\lambda_{1}^{F}, \xi_{1}^{F}, \xi_{2}^{F}, \lambda_{1}^{W}, \lambda_{2}^{W}, \xi_{1}^{W}$, $\xi_{2}^{W}$, and $L_{1}^{F}$. Substituting (B3) and (B5) into (B4), we have

$$
\begin{aligned}
& L_{2} H L_{1}^{F}\left[\frac{\partial W_{\text {stretch }}\left(\lambda_{1}^{F}, \xi_{1}^{F}, \xi_{1}^{F}\right)}{\partial \lambda_{1}^{F}}-\frac{P_{1}}{L_{2} H}-\varepsilon\left(\frac{\Phi}{H}\right)^{2} \lambda_{1}^{F}\left(\lambda_{2 p}\right)^{2}\right] \delta \lambda_{1}^{F}+L_{2} H L_{1}^{F} \frac{\partial W_{\text {stretch }}\left(\lambda_{1}^{F}, \xi_{1}^{F}, \xi_{2}^{F}\right)}{\partial \xi_{1}^{F}} \delta \xi_{1}^{F}+ \\
& L_{2} H L_{1}^{F} \frac{\partial W_{\text {stretch }}\left(\lambda_{1}^{F}, \xi_{1}^{F}, \xi_{2}^{F}\right)}{\partial \xi_{2}^{F}} \delta \xi_{2}^{F}+
\end{aligned}
$$




$$
\begin{aligned}
& L_{2} H L_{1}^{W}\left[\frac{\partial W_{\text {stretch }}\left(\lambda_{1}^{W}, \lambda_{2}^{W}, \xi_{1}^{W}, \xi_{2}^{W}\right)}{\partial \lambda_{1}^{W}}-\frac{P_{1}}{L_{2} H}-\varepsilon\left(\frac{\Phi}{H}\right)^{2} \lambda_{1}^{W}\left(\lambda_{2}^{W}\right)^{2}\right] \delta \lambda_{1}^{W}+ \\
& L_{2} H L_{1}^{W}\left[\frac{\partial W_{\text {stretch }}\left(\lambda_{1}^{W}, \lambda_{2}^{W}, \xi_{1}^{W}, \xi_{2}^{W}\right)}{\partial \lambda_{2}^{W}}-\varepsilon\left(\frac{\Phi}{H}\right)^{2}\left(\lambda_{1}^{W}\right)^{2} \lambda_{2}^{W}\right] \delta \lambda_{2}^{W}+L_{2} H L_{1}^{W} \frac{\partial W_{\text {stretch }}\left(\lambda_{1}^{W}, \lambda_{2}^{W}, \xi_{1}^{W}, \xi_{2}^{W}\right)}{\partial \xi_{1}^{W}} \delta \xi_{1}^{W}+ \\
& L_{2} H L_{1}^{W} \frac{\partial W_{\text {stretch }}\left(\lambda_{1}^{W}, \lambda_{2}^{W}, \xi_{1}^{W}, \xi_{2}^{W}\right)}{\partial \xi_{2}^{W}} \delta \xi_{2}^{W}+ \\
& L_{2} H\left[W_{\text {stretch }}\left(\lambda_{1}^{F}, \xi_{1}^{F}, \xi_{1}^{F}\right)-W_{\text {stretch }}\left(\lambda_{1}^{W}, \lambda_{2}^{W}, \xi_{1}^{W}, \xi_{2}^{W}\right)-\frac{\varepsilon}{2}\left(\frac{\Phi}{H}\right)^{2}\left(\lambda_{1}^{F} \lambda_{2 p}\right)^{2}+\frac{\varepsilon}{2}\left(\frac{\Phi}{H}\right)^{2}\left(\lambda_{1}^{W} \lambda_{2}^{W}\right)^{2}-\frac{P_{1}}{L_{2} H} \lambda_{1}^{F}+\frac{P_{1}}{L_{2} H} \lambda_{1}^{W}(\mathrm{~B} 6)^{F} \leq 0\right.
\end{aligned}
$$

The membrane is assumed to be in mechanical and electrostatic equilibrium. The coefficient in front of $\delta \lambda_{1}^{F}$ vanishes, recovering the equilibrium condition for the flat state given by (9) with $\lambda_{2}=\lambda_{2 p}$. The coefficients in front of $\delta \lambda_{1}^{W}$ and $\delta \lambda_{2}^{W}$ vanish, recovering the equilibrium conditions for the wrinkled state given by (9-10) with $\sigma_{2}=0$. The coefficient in front of $\delta L_{1}^{F}$ vanishes, giving the conditions for phase transition from the flat state to the wrinkled state

$$
W_{\text {stretch }}\left(\lambda_{1}^{F}, \xi_{1}^{F}, \xi_{2}^{F}\right)-\left(\frac{P_{1}}{L_{2} H}\right) \lambda_{1}^{F}-\frac{\varepsilon}{2}\left(\frac{\Phi}{H}\right)^{2}\left(\lambda_{1}^{F} \lambda_{2}^{F}\right)^{2}=W_{\text {stretch }}\left(\lambda_{1}^{W}, \lambda_{2}^{W}, \xi_{1}^{W}, \xi_{2}^{W}\right)-\left(\frac{P_{1}}{L_{2} H}\right) \lambda_{1}^{W}-\frac{\varepsilon}{2}\left(\frac{\Phi}{H}\right)^{2}\left(\lambda_{1}^{W} \lambda_{2}^{W}\right)^{2}
$$

Since the flat and wrinkled states have the same energy density, the flat and wrinkled regions can coexist during the discontinuous FW transition. In addition, the membrane is not in equilibrium with respect to viscoelastic relaxation, so that

$$
\begin{aligned}
& L_{2} H L_{1}^{F} \frac{\partial W_{\text {stretch }}\left(\lambda_{1}^{F}, \xi_{1}^{F}, \xi_{2}^{F}\right)}{\partial \xi_{1}^{F}} \delta \xi_{1}^{F}+L_{2} H L_{1}^{F} \frac{\partial W_{\text {stretch }}\left(\lambda_{1}^{F}, \xi_{1}^{F}, \xi_{2}^{F}\right)}{\partial \xi_{2}^{F}} \delta \xi_{2}^{F}+ \\
& L_{2} H L_{1}^{W} \frac{\partial W_{\text {stretch }}\left(\lambda_{1}^{W}, \lambda_{2}^{W}, \xi_{1}^{W}, \xi_{2}^{W}\right)}{\partial \xi_{1}^{W}} \delta \xi_{1}^{W}+L_{2} H L_{1}^{W} \frac{\partial W_{\text {stretch }}\left(\lambda_{1}^{W}, \lambda_{2}^{W}, \xi_{1}^{W}, \xi_{2}^{W}\right)}{\partial \xi_{2}^{W}} \delta \xi_{2}^{W} \leq 0
\end{aligned}
$$




\section{References}

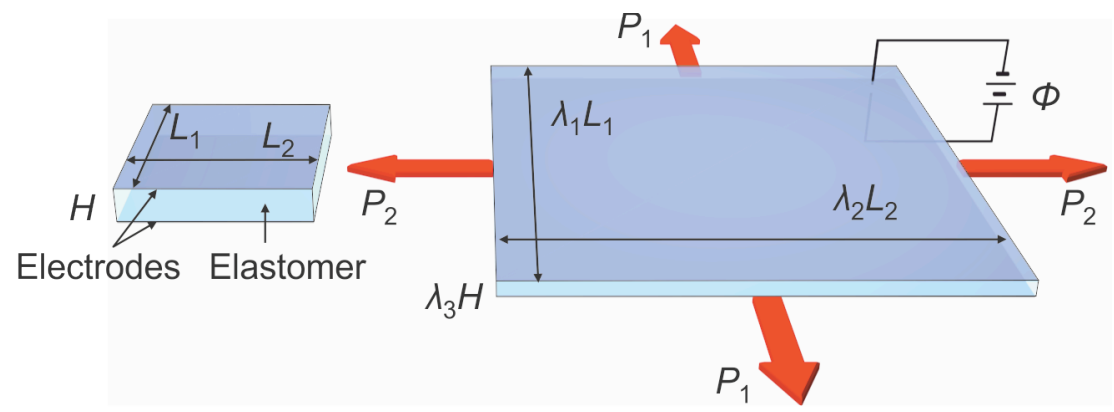

$\begin{array}{ll}\text { (a) Reference state } & \text { (b) Current state }\end{array}$

Fig. 1 A membrane of a dielectric elastomer, sandwiched between two compliant electrodes, is at (a) the reference state and (b) a current state. 

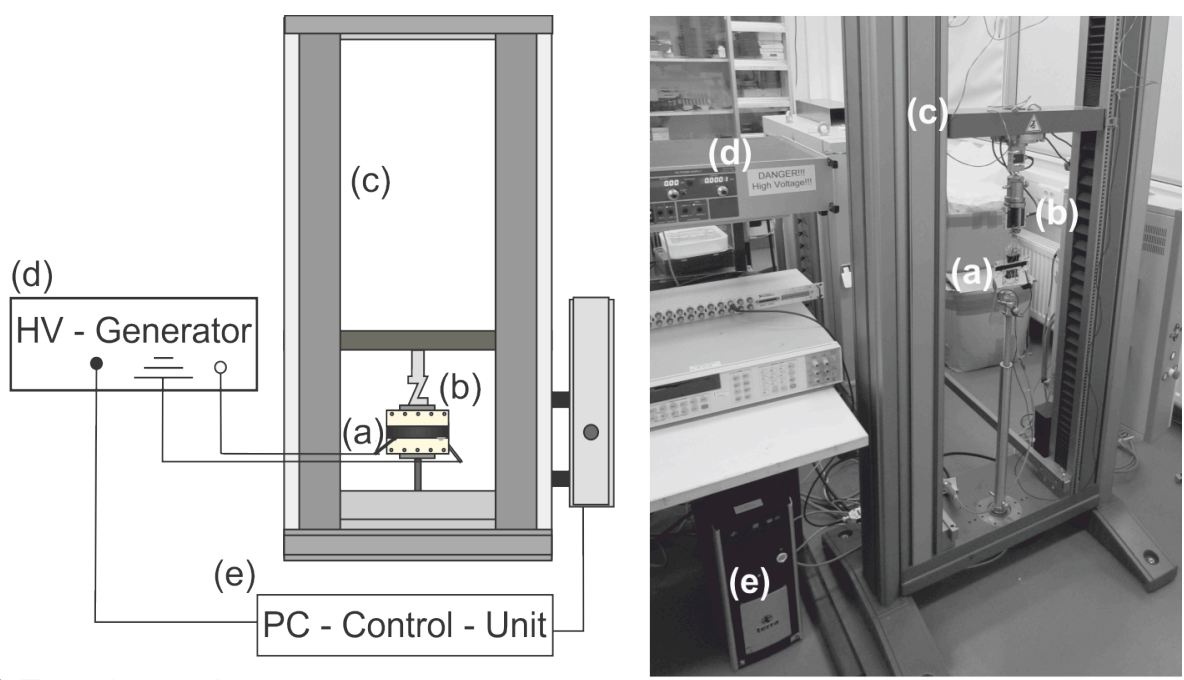

(1) Experimental setup
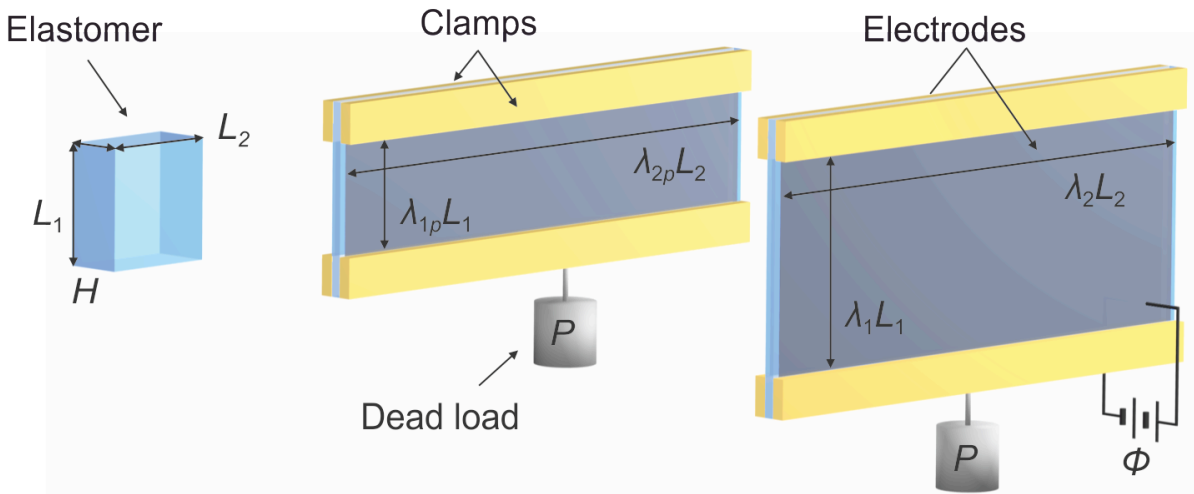

(2) Reference state

(3) Prestretched state

(4) Current state

Fig. 2 (1) The experimental setup consists of a clamped dielectric elastomer membrane $(a)$, an external strain gauge $(b)$, a frame structure $(c)$, and a high voltage generator $(d)$, which can be controlled by a PC (e). The dielectric elastomer membrane can be fabricated as follows. (2) The membrane is stress free. (3) The membrane is constrained by rigid clamps and subject to a force $\mathrm{P}$, and then coated with compliant electrodes on both surfaces. The membrane has stretches $\lambda_{1 p}$ and $\lambda_{2 p}$. (4) The membrane is subject to a voltage $\Phi$, and expands in the vertical direction. The membrane has stretches $\lambda_{1}$ and $\lambda_{2}$. 


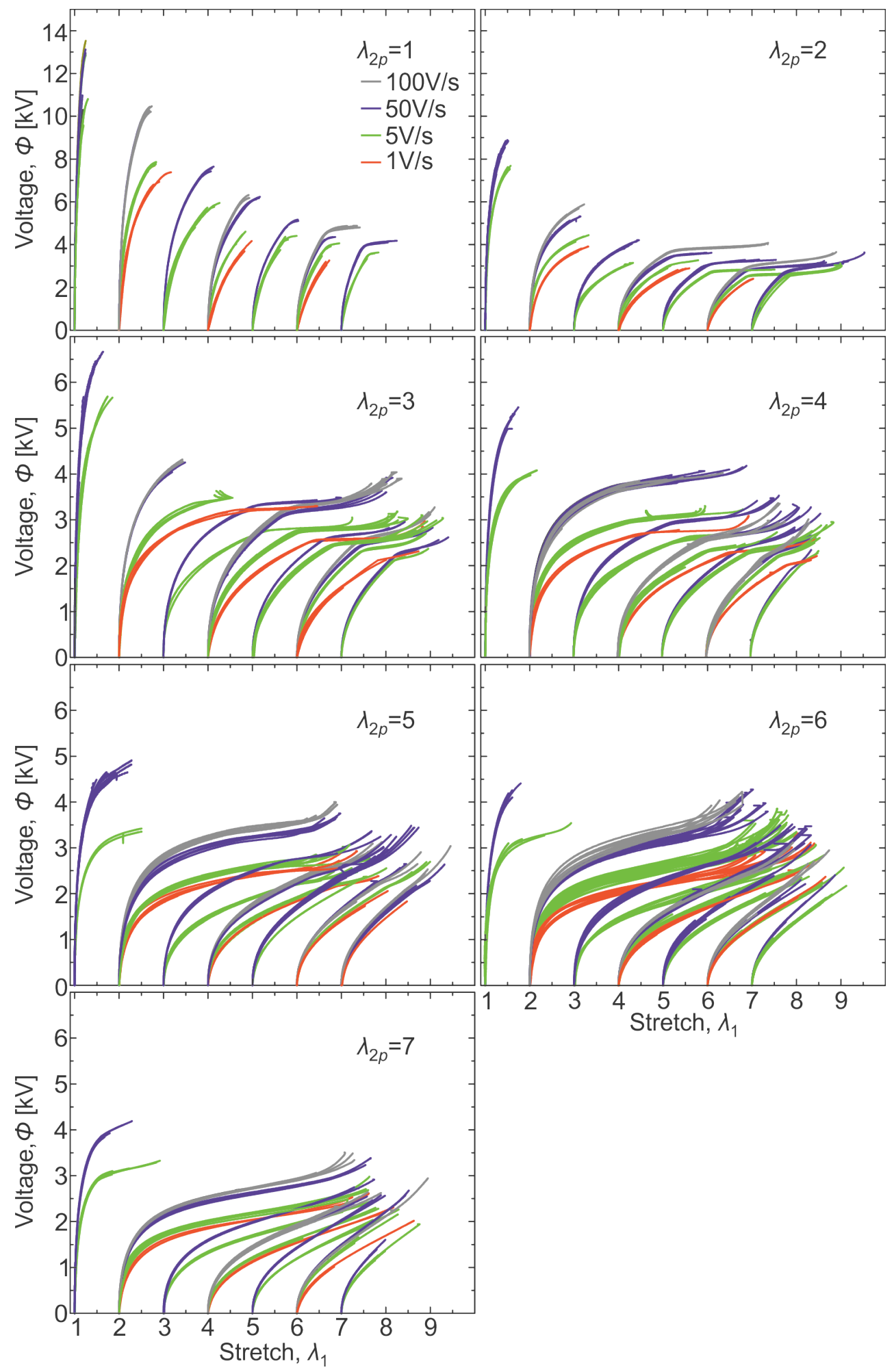

Fig. 3 The experimentally recorded voltage-stretch curves for clamped membranes in various states of prestretches $\left\{\lambda_{1 p}, \lambda_{2 p}\right\}$ at different voltage ramp rates. 


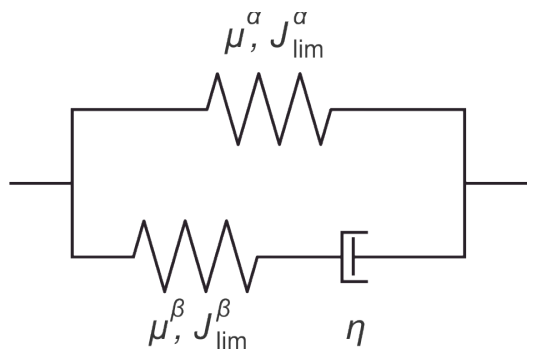

Fig. 4 A model of viscoelasticity for a membrane of a dielectric elastomer, which combines two units. One is an elastic spring ( ); the other is an elastic spring ( ) in series with a viscous dashpot. 


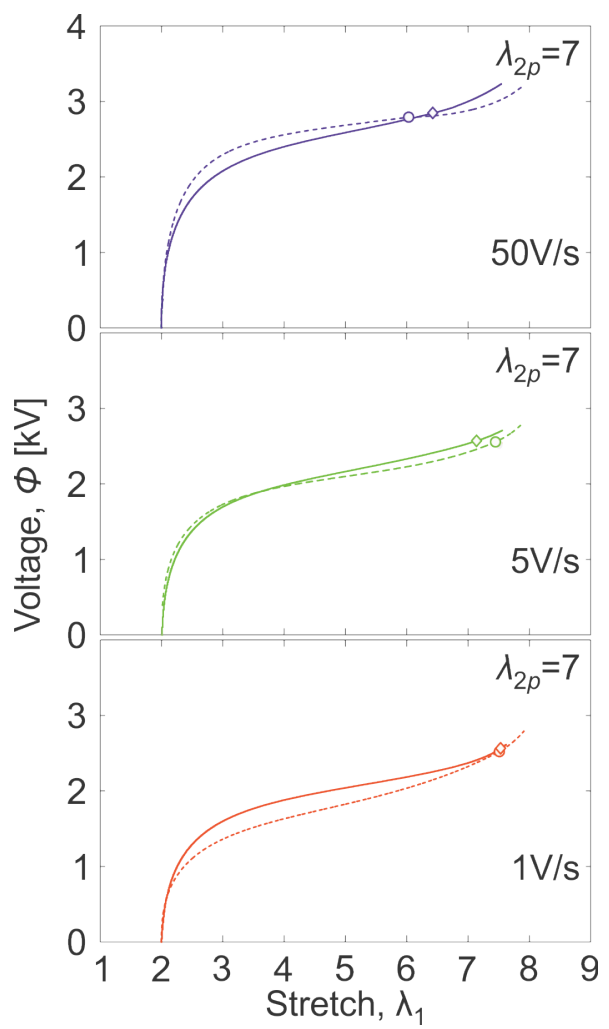

Fig. 5 Voltage-stretch curves for the membrane of prestretches $\{2,7\}$ at voltage ramp rates of $1 \mathrm{~V} / \mathrm{s}, 5 \mathrm{~V} / \mathrm{s}$ and $50 \mathrm{~V} / \mathrm{s}$. The solid curves represent the experimental data, while the dashed curves represent the theoretical calculations. The circles and diamonds represent the points at which the membrane suffers loss of tension in the theoretical predictions and experimentally observations, respetively. The material parameters used in the calculations are $\mu^{\alpha}=20 \mathrm{kPa}$, $\mu^{\beta}=200 \mathrm{kPa}, J_{\lim }^{\alpha}=155, J_{\lim }^{\beta}=155, \eta / \mu^{\beta}=50$, and $\varepsilon=4.12 \times 10^{-11} \mathrm{~F} / \mathrm{m}$. 


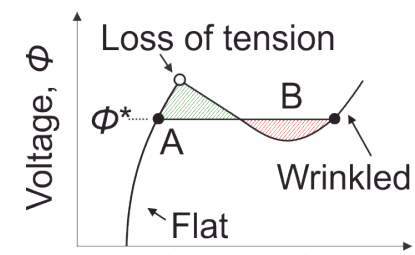

(a) Charge, $Q$

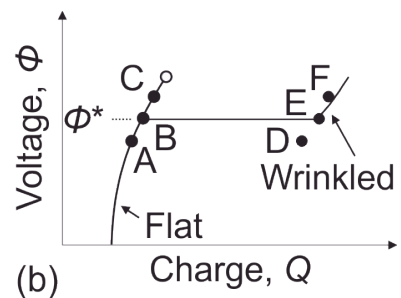

(b)

Fig. 6 The membrane exhibits a discontinuous transition from the flat state to the wrinkled state, and the flat and wrinkled regions can coexist at $\Phi^{*}$. (a) An elastic model is employed. The critical voltage $\Phi^{*}$ is at the level which makes two shaded regions have the equal area. (b) A viscoelastic model is employed. The critical voltage $\Phi^{*}$ is at the level which makes the flat and wrinkled states have the same energy density. 

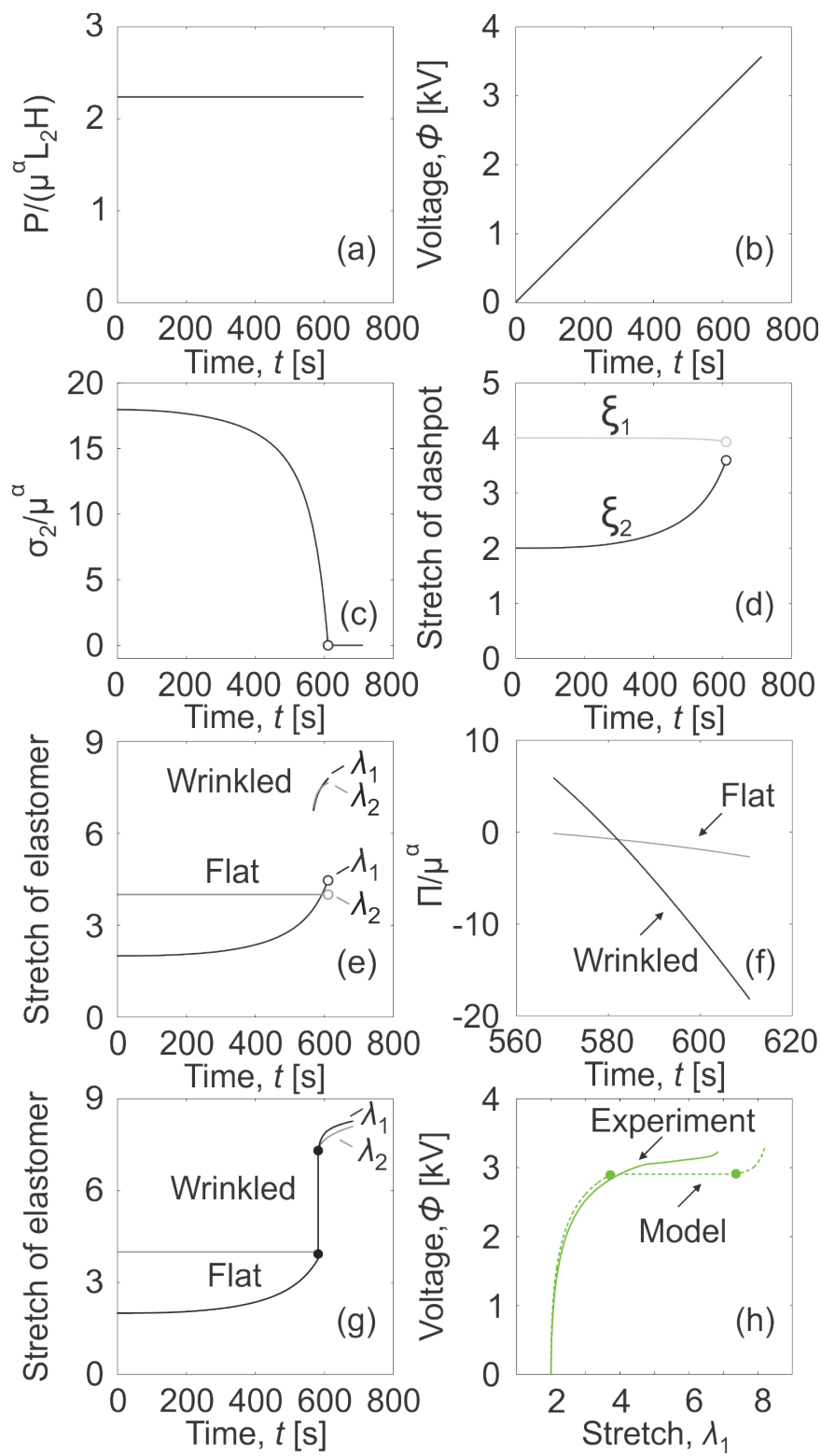

Fig. 7 Evolution and snap in the membrane of pre-stretches $\{2,4\}$ at a voltage ramp rate of 5 $\mathrm{V} / \mathrm{s}$. (a) The membrane is subject to a constant load in the vertical direction. (b) Voltage increases at a ramp rate of $5 \mathrm{~V} / \mathrm{s}$. (c) Horizontal tensile stress reduces as the voltage increases. At $t=613 \mathrm{~s}$, the horizontal tensile stress vanishes indicated by an open circle. (d) Evolution of the dashpot deformation. (e) Evolution of the elastomer deformation. When $t=568 \mathrm{~s}$, another wrinkled state of equilibrium exists. (f) Energy densities of the flat and wrinkled states. When $t$ $=582 \mathrm{~s}$, the two states have the same energy densities. (g) Evolution of the elastomer deformation. The snap through instability may occur at $t=582 \mathrm{~s}$. (h) Voltage-stretch curves. The solid curve represents the experimental data, while the dashed curve represents the theoretical predictions. At a certain voltage, the membrane transforms from the flat state to the wrinkled state, accompanied with a sudden snap in the vertical displacement indicated by filled circles. 

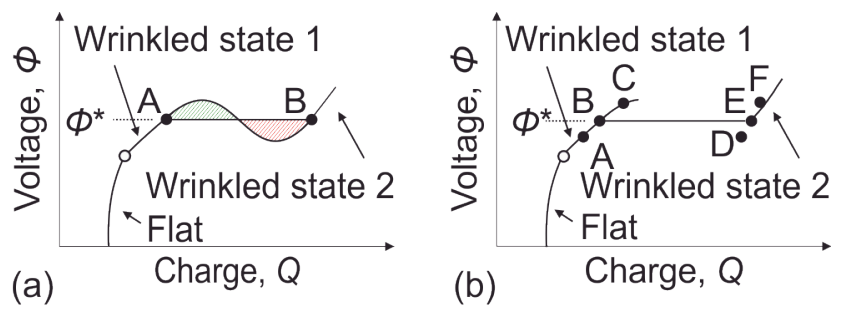

Fig. 8 Wrinkled-to-wrinkled transition. The membrane exhibits a continuous transition from the flat state to the wrinkled state, and then exhibits a discontinuous transition from the wrinkled state with a large wavelength to that with a small wavelength. (a) An elastic model is employed. The critical voltage $\Phi^{*}$ is at the level which makes two shaded regions have the equal area. (b) A viscoelastic model is employed. The critical voltage $\Phi^{*}$ is at the level which make wrinkled state 1 (with large wavelength) and wrinkled state 2 (with small wavelength) have same energy density. 

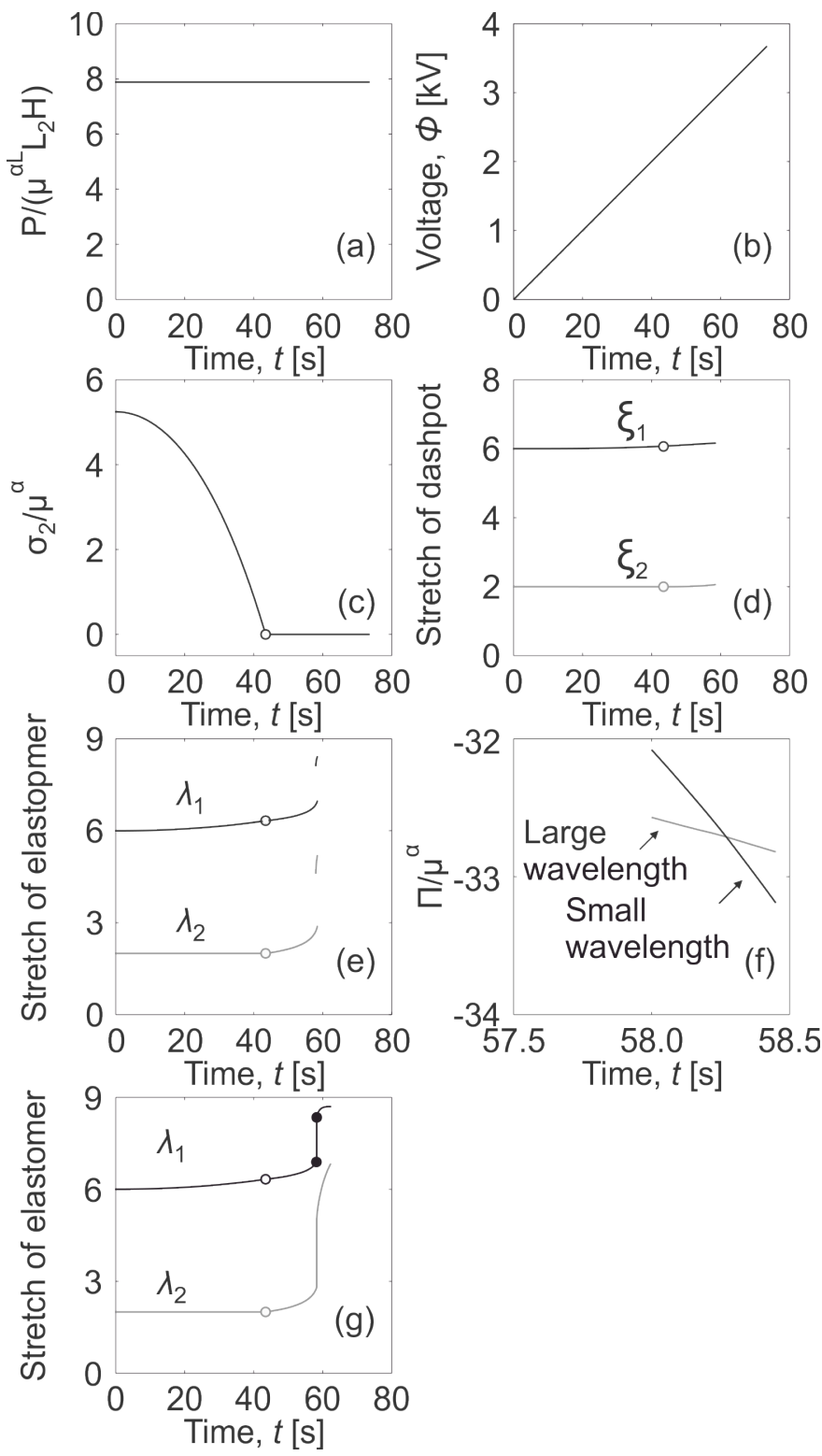

Fig. 9 Evolution and snap in the membrane of pre-stretches $\{6,2\}$ at a voltage ramp rate of 50 $\mathrm{V} / \mathrm{s}$. (a) The membrane is subject to a constant load in the vertical direction. (b) Voltage increases at a ramp rate of $50 \mathrm{~V} / \mathrm{s}$. (c) Horizontal tensile stress reduces as the voltage increases. At $t=43.4 \mathrm{~s}$, the horizontal tensile stress vanishes. The loss of tension is indicated as open circles and causes the membrane to form wrinkles. (d) Evolution of the dashpot deformation. (e) Evolution of the elastomer deformation. When $t=58 \mathrm{~s}$, another wrinkled state of equilibrium exists with a small wavelength. (f) Energy densities of the two wrinkled states. One has a small wavelength; the other has a large wavelength. When $t=58.3 \mathrm{~s}$, the two wrinkled states have the 
same energy density. (g) Evolution of the elastomer deformation. The membrane exhibits a continuous transition from the flat state to the wrinkled state at $t=43.4 \mathrm{~s}$, and then exhibits a discontinuous transition (indicated by solid circles) from the wrinkled state with a large wavelength to that with a small wavelength at $t=58.3 \mathrm{~s}$. 

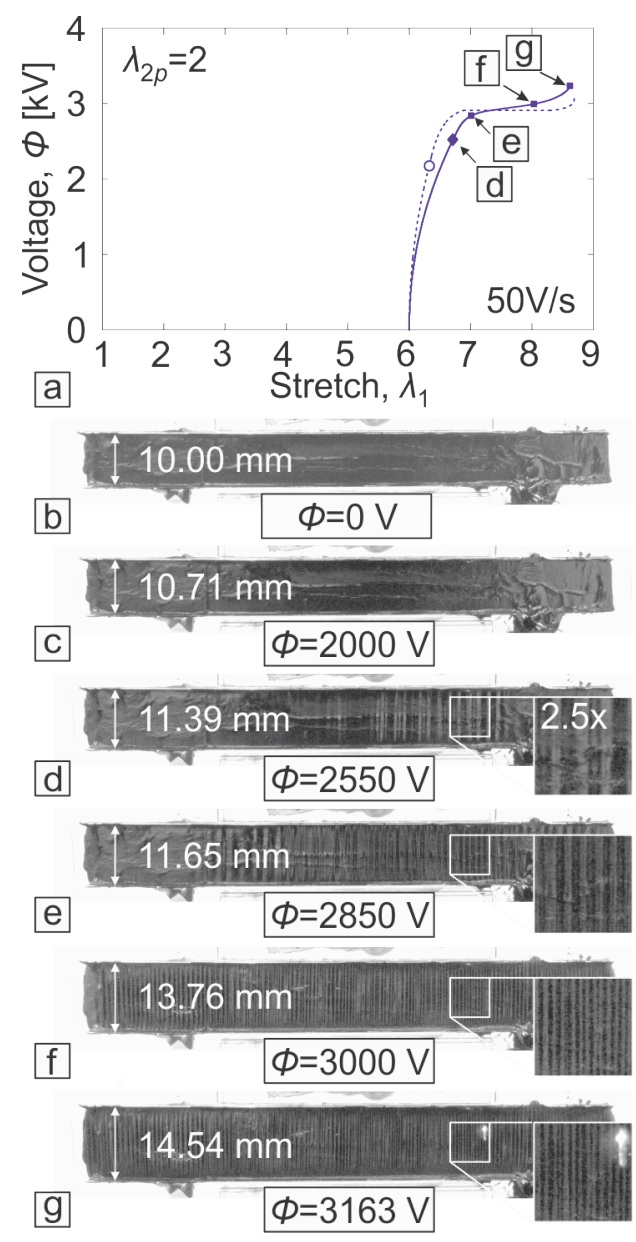

Fig. 10 (a) Voltage-stretch curves for the membrane of prestretches $\{6,2\}$ at a voltage ramp rate of $50 \mathrm{~V} / \mathrm{s}$. The solid curve represents the experimental data, while the dashed curve represents the theoretical predictions. The open circle indicates the calculated loss of tension while the diamond notifies the experimental observation respectively. The membrane exhibits a continuous transition from the flat state to the wrinkled state, and then exhibits a discontinuous transition from the wrinkled state with a large wavelength to that with a small wavelength. (b-g) Experimental observations. (b) The membrane is flat, subject to no voltage. (c) The membrane is still flat, when the voltage is small. (d) The membrane suffers loss of tension, and exhibits a continuous transition from the flat state to the wrinkled state at $\Phi=2550$ V. (e-f) The membrane suffers snap through instability at $\Phi=2850 \mathrm{~V}$, and exhibits a discontinuous transition from the 
wrinkled state with a large wavelength to that with a small wavelength within $3 \mathrm{~s}$. (g) The membrane suffers electric breakdown at $\Phi=3163 \mathrm{~V}$. 


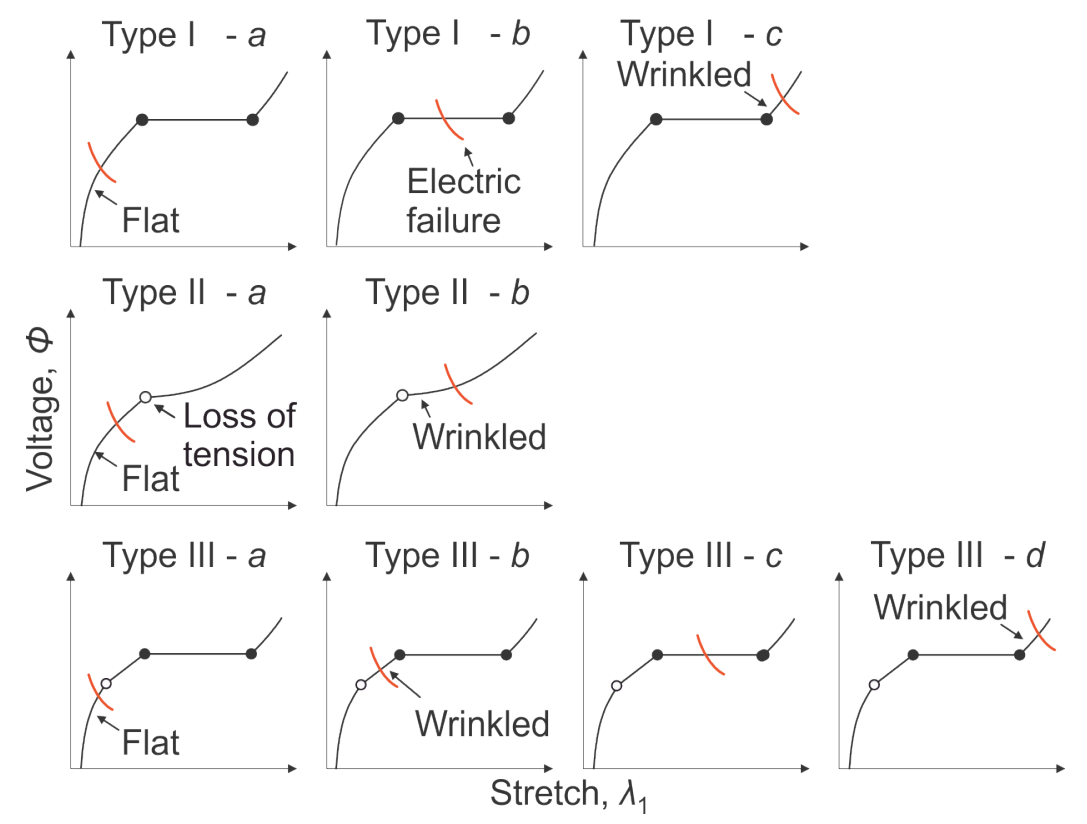

Fig. 11 Representative electromechanical behavior of a clamped membrane. The black curve represents the states of equilibrium, while the red curve represents the condition of dielectric breakdown. The open circle represents that the membrane suffers loss of tension and forms wrinkles, and the two solid circles represent the two states that have the same energy density and can coexist. We identify three types of transition, according to the black curve which may combine loss of tension and snap through instability. We identify nine types of behavior, according to where the red and black curves intersect. 


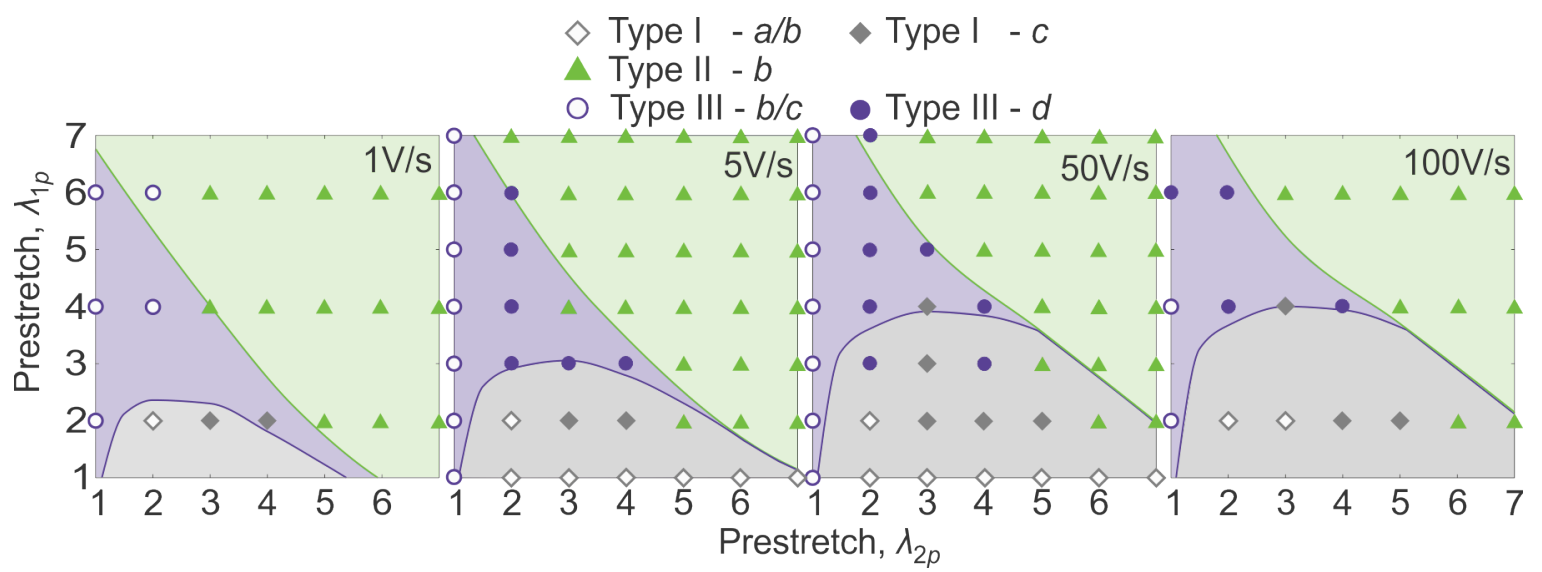

Fig. 12 The clamped membrane of a dielectric elastomer shows different kinds of behavior, depending on pre-stretches and voltage ramp rates. The theory predicts that the membrane exhibits Type I transition in the grey area, a Type II transition in the green area and a Type III transition in the blue area. The diamonds, triangles and circles corresponds to the dominant experimental observations of expected electromechanical behavior sketched in Fig. 11. 

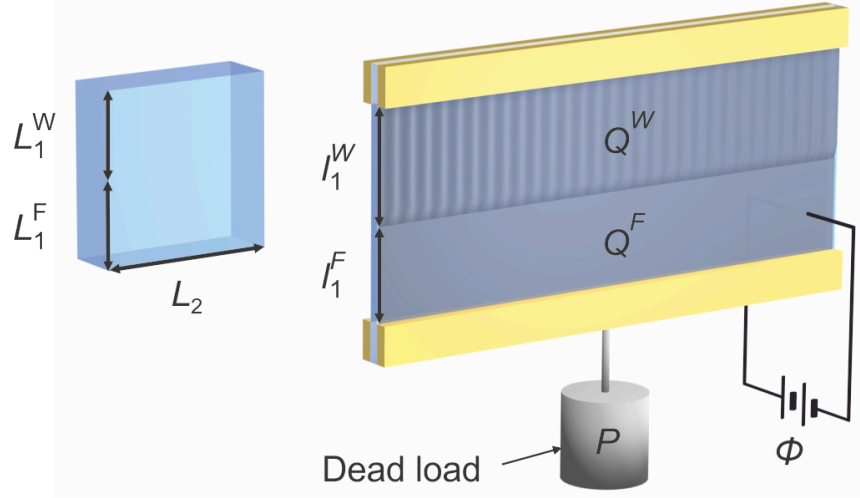

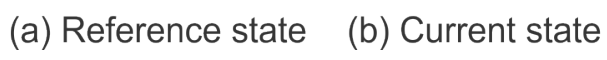

Fig. B1 During a discontinuous phase transition, the flat and wrinkled regions can coexist. (a) In the reference state, the flat and wrinkled regions are of lengths $L_{1}^{F}$ and $L_{1}^{W}$, respectively. (b) In the current state, the flat and wrinkled regions are of lengths $l_{1}^{F}$ and $l_{1}^{W}$ and charges $Q^{F}$ and $Q^{W}$ 